\title{
THE SASAKI CONE AND EXTREMAL SASAKIAN METRICS
}

\author{
CHARLES P. BOYER, KRZYSZTOF GALICKI, AND SANTIAGO R. SIMANCA
}

\begin{abstract}
We study the Sasaki cone of a CR structure of Sasaki type on a given closed manifold. We introduce an energy functional over the cone, and use its critical points to single out the strongly extremal Reeb vectors fields. Should one such vector field be a member of the extremal set, the scalar curvature of a Sasaki extremal metric representing it would have the smallest $L^{2}$-norm among all Sasakian metrics of fixed volume that can represent vector fields in the cone. We use links of isolated hypersurface singularities to produce examples of manifolds of Sasaki type, many of these in dimension five, whose Sasaki cone coincides with the extremal set, and examples where the extremal set is empty. We end up by proving that a conjecture of Orlik concerning the torsion of the homology groups of these links holds in the five dimensional case.
\end{abstract}

\section{INTRODUCTION}

The study of a Sasakian structure goes along with the one dimensional foliation on the manifold associated to the Reeb vector field. In the case where the orbits are all closed, the manifold has the structure of an orbifold circle bundle over a compact Kähler orbifold, which must be algebraic, and which has at most cyclic quotient singularities. For a long time, techniques from Riemannian submersions, suitably extended, were used to find interesting canonical Sasakian metrics in this orbibundle setting. This led to the common belief that the only interesting such metrics occurred precisely in this setting. Compact Sasakian manifolds with nonclosed leaves - irregular Sasakian structures - were known to exist, but there was no evidence to suspect they could be had with Einstein metrics as well. It was thought reasonable that all Sasaki-Einstein metrics could be understood well by simply studying the existence of Kähler-Einstein metrics on compact cyclic orbifolds.

The existence of Sasaki-Einstein metrics is of great interest in the physics of the famous CFT/AdS Duality Conjecture, and the discovery of irregular SasakiEinstein structures [18] was a rather remarkable feat, in particular if we take into account their rather explicit depiction in coordinates. An attempt to study them using a treatment inspired in the use of classical Lagrangians, replaced in this context by a Riemannian functional whose critical points define the canonical metrics we seek, was started in [13] using the squared $L^{2}$-norm of the scalar curvature as the functional in question, with its domain restricted to the space of metrics adapted to the geometry of the underlying Sasakian structure under consideration. This choice of Lagrangian is well known in Kähler geometry, which was and remains a guiding principle to us, and is quite natural since Sasakian geometry sits naturally

During the preparation of this work, the first two authors were partially supported by NSF grant DMS-0504367. 
in between two Kähler geometries, that of the transversals to the one dimensional foliations associated to the Reeb vector field, and that of the metric cones inside which the Sasaki manifolds sits as a base. Not surprisingly, the Sasakian metrics that are critical points of the Lagrangian we use are those that are transversally extremal in the sense of Calabi, and several important results known in Kähler geometry have now a counterpart in the Sasakian context [13, 17] also.

In the theory we developed 13 , a given CR structure $(\mathcal{D}, J)$ of Sasaki type is the analogue in the Sasaki context of a complex structure on a manifold of Kähler type, and the Reeb vector field $\xi$ in a Sasakian structure with $(\mathcal{D}, J)$ as underlying CR structure is the analogue of a choice of a class in the Kähler cone. The Sasaki cone $\kappa(\mathcal{D}, J)$ of the $\mathrm{CR}$ structure arises naturally, and $\xi \in \kappa(\mathcal{D}, J)$ is said to be canonical or extremal if there exists a Sasakian structure $(\xi, \eta, \Phi, g)$, with underlying CR structure $(\mathcal{D}, J)$, for which the metric $g$ in the structure is Sasaki extremal. The set of vector fields in the cone for which this happens is denoted by $\mathfrak{e}(\mathcal{D}, J)$. In this article we present a number of examples where $\kappa(\mathcal{D}, J)=\mathfrak{e}(\mathcal{D}, J)$, as well as a number of examples where $\mathfrak{e}(\mathcal{D}, J)$ is the empty set.

In the cases where $\kappa(\mathcal{D}, J)=\mathfrak{e}(\mathcal{D}, J)$, it is natural to ask which is the optimal choice we could make of a Reeb vector field, be as it may that each one admits a Sasaki extremal representative. In effect, this question turns out to be natural in general, even in the cases where $\mathfrak{e}(\mathcal{D}, J)$ is the empty set. We briefly discuss this here also, introducing the notion of strongly extremal Reeb vector field. A detailed elaboration of this idea will appear elsewhere.

The specific examples we give are links of isolated hypersurface singularities. For these manifolds, there is a long standing conjecture of Orlik whose validity would describe the torsion of their homology groups. We present a proof of this conjecture in dimension five, as obtained recently by the second author.

This article developed from partial results obtained by the three authors while working on an ongoing project. It is presented here by the first and last author in memory of their friend and collaborator KRIS GALICKI.

\section{Preliminaries on SASAKIAN GeOMEtry}

A contact metric structure $(\xi, \eta, \Phi, g)$ on a manifold $M$ is said to be a Sasakian structure if $(\xi, \eta, \Phi)$ is normal. A smooth manifold provided with one such structure is said to be a Sasakian manifold, or a manifold of Sasaki type. We briefly spell out the meaning of this definition in order to recall the relevant geometric set-up it brings about. A detailed discussion of Sasakian and contact geometry can be found in [7].

The triple $(\xi, \eta, \Phi)$ defines an almost contact structure on $M$. That is to say, $\xi$ is a nowhere zero vector field, $\eta$ is a one form, and $\Phi$ is a tensor of type $(1,1)$, such that

$$
\eta(\xi)=1, \quad \Phi^{2}=-11+\xi \otimes \eta .
$$

The vector field $\xi$ defines the characteristic foliation $\mathcal{F}_{\xi}$ with one-dimensional leaves, and the kernel of $\eta$ defines the codimension one sub-bundle $\mathcal{D}$. We have the canonical splitting

$$
T M=\mathcal{D} \oplus L_{\xi}
$$

where $L_{\xi}$ is the trivial line bundle generated by $\xi$. 
The sub-bundle $\mathcal{D}$ inherits an almost complex structure $J$ by restriction of $\Phi$, and the dimension of $M$ must be an odd integer, which we set be $2 n+1$ here. When forgetting the tensor $\Phi$ and characteristic foliation, the sub-bundle $\mathcal{D}$ by itself defines what is called a contact structure on $M$.

The Riemannian metric $g$ is compatible with the almost contact structure $(\xi, \eta, \Phi)$, and so we have have that

$$
g(\Phi(X), \Phi(Y))=g(X, Y)-\eta(X) \eta(Y) .
$$

Thus, $g$ induces an almost Hermitian metric on $\mathcal{D}$. This fact makes of $(\xi, \eta, \Phi, g)$ an almost contact metric structure, and in that case, the canonical decomposition (11) is orthogonal. The orbits of the field $\xi$ are geodesics, a condition that can be re-expressed as $\xi \downarrow d \eta=0$, and so $d \eta$ is a basic 2 -form. We have the relation

$$
g(\Phi X, Y)=d \eta(X, Y)
$$

which says that $(\xi, \eta, \Phi, g)$ is a contact metric structure. Notice that the volume element defined by $g$ is given by

$$
d \mu_{g}=\frac{1}{n !} \eta \wedge(d \eta)^{n} .
$$

On the cone $C(M)=M \times \mathbb{R}^{+}$we have the metric

$$
g_{C}=d r^{2}+r^{2} g
$$

and the radial vector field $r \partial_{r}$ satisfies the relation

$$
£_{r \partial_{r}} g_{C}=2 g_{C} \text {. }
$$

We have also an almost complex structure $I$ on $C(M)$ given by

$$
I(Y)=\Phi(Y)-\eta(Y) r \partial_{r}, \quad I\left(r \partial_{r}\right)=\xi .
$$

The contact metric structure $(\xi, \eta, \Phi)$ is said to be normal if the pair $(C(M), I)$ is a complex manifold. In that case, the induced almost complex structure $J$ on $\mathcal{D}$ is integrable. Further, $g_{C}$ is a Kähler metric on $(C(M), I)$.

2.1. Transverse Kähler structure. For a Sasakian structure $(\xi, \eta, \Phi, g)$, the integrability of the almost complex structure $I$ on the cone $C(M)$ implies that the Reeb vector field $\xi$ leaves both, $\eta$ and $\Phi$, invariant. We obtain a codimension one integrable strictly pseudo-convex CR structure $(\mathcal{D}, J)$, where $\mathcal{D}=\operatorname{ker} \eta$ is the contact bundle and $J=\left.\Phi\right|_{\mathcal{D}}$, and the restriction of $g$ to $\mathcal{D}$ defines a symmetric form on $(\mathcal{D}, J)$ that we shall refer to as the transverse Kähler metric $g^{T}$. By (2), the Kähler form of the transverse Kähler metric is given by the form $d \eta$. Therefore, the Sasakian metric $g$ is determined fully in terms of $(\xi, \eta, \Phi)$ by the expression

$$
g=d \eta \circ(\mathbb{1} \otimes \Phi)+\eta \otimes \eta .
$$

The Killing field $\xi$ leaves invariant $\eta$ and $\Phi$, and the decomposition (1) is orthogonal. Despite its dependence on the other elements of the structure, we insist on explicitly referring to $g$ as part of the Sasakian structure $(\xi, \eta, \Phi, g)$.

We consider the set

$$
\mathcal{S}(\xi)=\{\text { Sasakian structure }(\tilde{\xi}, \tilde{\eta}, \tilde{\Phi}, \tilde{g}) \mid \tilde{\xi}=\xi\},
$$

and provide it with the $C^{\infty}$ compact-open topology as sections of vector bundles. For any element $(\tilde{\xi}, \tilde{\eta}, \tilde{\Phi}, \tilde{g})$ in this set, the 1 -form $\zeta=\tilde{\eta}-\eta$ is basic, and so $[d \tilde{\eta}]_{B}=[d \eta]_{B}$. Here, $[\cdot]_{B}$ stands for a cohomology class in the basic cohomology 
ring, a ring that is defined by the restriction $d_{B}$ of the exterior derivative $d$ to the subcomplex of basic forms in the de Rham complex of $M$. Thus, all of the Sasakian structures in $\mathcal{S}(\xi)$ correspond to the same basic cohomology class. We call $\mathcal{S}(\xi)$ the space of Sasakian structures compatible with $\xi$, and say that the Reeb vector field $\xi$ polarizes the Sasakian manifold $M[13$.

Given a Reeb vector field $\xi$, we have its characteristic foliation $\mathcal{F}_{\xi}$, so we let $\nu\left(\mathcal{F}_{\xi}\right)$ be the vector bundle whose fiber at a point $p \in M$ is the quotient space $T_{p} M / L_{\xi}$, and let $\pi_{\nu}: T M \rightarrow \nu\left(\mathcal{F}_{\xi}\right)$ be the natural projection. The background structure $\mathcal{S}=(\xi, \eta, \Phi, g)$ induces a complex structure $\bar{J}$ on $\nu\left(\mathcal{F}_{\xi}\right)$. This is defined by $\bar{J} \bar{X}:=\overline{\Phi(X)}$, where $X$ is any vector field in $M$ such that $\pi(X)=\bar{X}$. Furthermore, the underlying CR structure $(\mathcal{D}, J)$ of $\mathcal{S}$ is isomorphic to $\left(\nu\left(\mathcal{F}_{\xi}\right), \bar{J}\right)$ as a complex vector bundle. For this reason, we refer to $\left(\nu\left(\mathcal{F}_{\xi}\right), \bar{J}\right)$ as the complex normal bundle of the Reeb vector field $\xi$, although its identification with $(\mathcal{D}, J)$ is not canonical. In this sense, $M$ is polarized by $(\xi, \bar{J})$.

We define $\mathcal{S}(\xi, \bar{J})$ to be the subset of all structures $(\tilde{\xi}, \tilde{\eta}, \tilde{\Phi}, \tilde{g})$ in $\mathcal{S}(\xi)$ such that the diagram

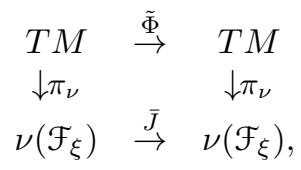

commutes. This set consists of elements of $\mathcal{S}(\xi)$ with the same transverse holomorphic structure $\bar{J}$, or with more precision, the same complex normal bundle $\left(\nu\left(\mathcal{F}_{\xi}\right), \bar{J}\right)$.

2.2. The Sasaki cone. If we look at the Sasakian structure $(\xi, \eta, \Phi, g)$ from the point of view of CR geometry, its underlying strictly pseudo-convex CR structure $(\mathcal{D}, J)$, with associated contact bundle $\mathcal{D}$, has Levi form $d \eta$.

If $(\mathcal{D}, J)$ is a strictly pseudo-convex $\mathrm{CR}$ structure on $M$ of codimension one, we say that $(\mathcal{D}, J)$ is of Sasaki type if there exists a Sasakian structure $\mathcal{S}=(\xi, \eta, \Phi, g)$ such that $\mathcal{D}=\operatorname{ker} \eta$ and $\left.\Phi\right|_{\mathcal{D}}=J$. We consider the set

$$
\mathcal{S}(\mathcal{D}, J)=\left\{\begin{array}{c}
\mathcal{S}=(\xi, \eta, \Phi, g): \mathcal{S} \text { a Sasakian structure } \\
(\operatorname{ker} \eta, \Phi \mid \operatorname{ker} \eta)=(\mathcal{D}, J)
\end{array}\right\}
$$

of Sasakian structures with underlying CR structure $(\mathcal{D}, J)$.

We denote by $\operatorname{con}(\mathcal{D})$ the Lie algebra of infinitesimal contact transformations, and by $\mathfrak{c r}(\mathcal{D}, J)$ the Lie algebra of the group $\mathfrak{C} \mathfrak{R}(\mathcal{D}, J)$ of CR automorphism of $(\mathcal{D}, J)$. If $\mathcal{S}=(\xi, \eta, \Phi, g)$ is a contact metric structure whose underlying CR structure is $(\mathcal{D}, J)$, then $\mathcal{S}$ is a Sasakian structure if, and only if, $\xi \in \mathfrak{c r}(\mathcal{D}, J)$.

The vector field $X$ is said to be positive for $(\mathcal{D}, J)$ if $\eta(X)>0$ for any $(\xi, \eta, \Phi, g) \in$ $\mathcal{S}(\mathcal{D}, J)$. We denote by $\mathfrak{c r}^{+}(\mathcal{D}, J)$ the subset of all of these positive fields, and consider the projection

$$
\begin{array}{llc}
\mathcal{S}(\mathcal{D}, J) & \stackrel{\iota}{ } & \mathfrak{c r}^{+}(\mathcal{D}, J) \\
(\xi, \eta, \Phi, g) & \mapsto & \xi
\end{array} .
$$

This mapping identifies naturally $\mathfrak{c r}^{+}(\mathcal{D}, J)$ with $\mathcal{S}(\mathcal{D}, J)$. Furthermore, $\mathfrak{c r}^{+}(\mathcal{D}, J)$ is an open convex cone in $\mathfrak{c r}(\mathcal{D}, J)$ that is invariant under the adjoint action of the Lie group $\mathfrak{C} \mathfrak{R}(\mathcal{D}, J)$. The identification (8) gives the moduli space $\mathcal{S}(\mathcal{D}, J) / \mathfrak{C} \mathfrak{R}(\mathcal{D}, J)$ of all Sasakian structures whose underlying CR structure is $(\mathcal{D}, J)$. The conical structure of $\mathfrak{c r}^{+}(\mathcal{D}, J)$ justifies the following definition: 
Definition 2.1. Let $(\mathcal{D}, J)$ be a $\mathrm{CR}$ structure of Sasaki type on $M$. The Sasaki cone $\kappa(\mathcal{D}, J)$ is the moduli space of Sasakian structures compatible with $(\mathcal{D}, J)$,

$$
\kappa(\mathcal{D}, J)=\mathcal{S}(\mathcal{D}, J) / \mathfrak{C} R(\mathcal{D}, J) .
$$

The isotropy subgroup of an element $\mathcal{S} \in \mathcal{S}(\mathcal{D}, J)$ is, by definition, $\mathfrak{A} \mathfrak{u t}(\mathcal{S})$. It contains a maximal torus $T_{k}$. In fact, we have the following result [13]:

Theorem 2.2. Let $M$ be a closed manifold of dimension $2 n+1$, and let $(\mathcal{D}, J)$ be a CR structure of Sasaki type on it. Then the Lie algebra $\mathfrak{c r}(\mathcal{D}, J)$ decomposes as $\mathfrak{c r}(\mathcal{D}, J)=\mathfrak{t}_{k}+\mathfrak{p}$, where $\mathfrak{t}_{k}$ is the Lie algebra of a maximal torus $T_{k}$ of dimension $k, 1 \leq k \leq n+1$, and $\mathfrak{p}$ is a completely reducible $T_{k}$-module. Furthermore, every $X \in \mathfrak{c r}^{+}(\mathcal{D}, J)$ is conjugate to a positive element in the Lie algebra $\mathfrak{t}_{k}$.

Theorem 2.2 and (8) imply that each orbit can be represented by choosing a positive element in the Lie algebra $\mathfrak{t}_{k}$ of a maximal torus $T_{k}$. So let us fix a maximal torus $T_{k}$ of a maximal compact subgroup $G$ of $\mathfrak{C R}(\mathcal{D}, J)$, and let $\mathcal{W}$ denote the Weyl group of $G$. If $\mathfrak{t}_{k}^{+}=\mathfrak{t}_{k} \cap \mathfrak{c r}^{+}(\mathcal{D}, J)$ denotes the subset of positive elements in $\mathfrak{t}_{k}$, we have the identification $\kappa(\mathcal{D}, J)=\mathfrak{t}_{k}^{+} / \mathcal{W}$. Each Reeb vector field in $\mathfrak{t}_{k}^{+} / \mathcal{W}$ corresponds to a unique Sasakian structure, so we can view $\mathfrak{t}_{k}^{+} / \mathcal{W}$ as a subset of $\mathcal{S}$, and we have

Lemma 2.3. Let $(\mathcal{D}, J)$ be a $\mathrm{CR}$ structure of Sasaki type on $M$, and fix a maximal torus $T_{k} \in \mathfrak{C} \mathfrak{R}(\mathcal{D}, J)$. Then we have that

$$
\bigcap_{\mathcal{S} \in \mathfrak{t}_{k}^{+} / \mathcal{W}} \mathfrak{A u t}_{0}(\mathcal{S})=T_{k},
$$

where $\mathfrak{A u t}_{0}(\mathcal{S})$ denotes the identity component of the isotropy group $\mathfrak{A} \mathfrak{u t}(\mathcal{S})$. In particular, $T_{k}$ is contained in the isotropy subgroup of every $\mathcal{S} \in \mathfrak{t}_{k}^{+} / \mathcal{W}$.

Now the basic Chern class of a Sasakian structure $\mathcal{S}=(\xi, \eta, \Phi, g)$ is represented by the Ricci form $\rho^{T} / 2 \pi$ of the transverse metric $g_{T}$. Although the notion of basic changes with the Reeb vector field, the complex vector bundle $\mathcal{D}$ remains fixed. Hence, for any Sasakian structure $\mathcal{S} \in \mathcal{S}(\mathcal{D}, J)$, the transverse 2 -form $\rho^{T} / 2 \pi$ associated to $\mathcal{S}$ represents the first Chern class $c_{1}(\mathcal{D})$ of the complex vector bundle $\mathcal{D}$.

When $k=1$, the maximal torus of $\mathfrak{C} \mathfrak{R}(\mathcal{D}, J)$ is one dimensional. Since the Reeb vector field is central, this implies that $\operatorname{dim} \mathfrak{a u t}(\mathcal{S})=\operatorname{dim} \mathfrak{c r}(\mathcal{D}, J)=1$. Hence, we have that $\mathcal{S}(\mathcal{D}, J)=\mathfrak{c r}^{+}(\mathcal{D}, J)=\mathfrak{t}_{1}^{+}=\mathbb{R}^{+}$, and $\mathcal{S}(\mathcal{D}, J)$ consists of the 1-parameter family of Sasakian structures given by $\mathcal{S}_{a}=\left(\xi_{a}, \eta_{a}, \Phi_{a}, g_{a}\right)$, where

$$
\xi_{a}=a^{-1} \xi, \quad \eta_{a}=a \eta, \quad \Phi_{a}=\Phi, \quad g_{a}=a g+\left(a^{2}-a\right) \eta \otimes \eta,
$$

and $a \in \mathbb{R}^{+}$, the 1-parameter family of transverse homotheties.

In effect, the transverse homotheties (9) are the only deformations $\left(\xi_{t}, \eta_{t}, \Phi_{t}, g_{t}\right)$ of a given structure $\mathcal{S}=(\xi, \eta, \Phi, g)$ in the Sasakian cone $\kappa(\mathcal{D}, J)$ where the Reeb vector field varies in the form $\xi_{t}=f_{t} \xi, f_{t}$ a scalar function. For we then have that the family of tensors $\Phi_{t}$ is constant, and since $£_{\xi_{t}} \Phi_{t}=0$, we see that $f_{t}$ must be annihilated by any section of the sub-bundle $\mathcal{D}$. But then (2) implies that $d f_{t}=\left(\xi f_{t}\right) \eta$, and we conclude that the function $f_{t}$ is constant. Thus, in describing fully the tangent space of $\mathcal{S}(\mathcal{D}, J)$ at $\mathcal{S}$, it suffices to describe only those deformations $\left(\xi_{t}, \eta_{t}, \Phi_{t}, g_{t}\right)$ where $\dot{\xi}=\left.\partial_{t} \xi_{t}\right|_{t=0}$ is $g$-orthogonal to $\xi$. These correspond to 
deformations where the volume of $M$ in the metric $g_{t}$ remains constant in $t$, and are parametrized by elements of $\kappa(\mathcal{D}, J)$ that are $g$-orthogonal to $\xi$.

The terminology we use here is chosen to emphasize the analogy that the Sasaki cone is to a CR structure of Sasaki type what the Kähler cone is to a complex manifold of Kähler type. Indeed, for any point $\mathcal{S}=(\xi, \eta, \Phi, g)$ in $\kappa(\mathcal{D}, J)$, the complex normal bundle $\left(\nu\left(\mathfrak{F}_{\xi}\right), \bar{J}\right)$ is isomorphic to $(\mathcal{D}, J)$. In this sense, the complex structure $\bar{J}$ is fixed with the fixing of $(\mathcal{D}, J)$, the Reeb vector field $\xi$ polarizes the manifold, and the Sasaki cone $\kappa(\mathcal{D}, J)$ represents the set of all possible polarizations. We observe though that when we fix $\xi \in \kappa(\mathcal{D}, J)$, the underlying CR structure of elements in $\mathcal{S}(\xi, \bar{J})$ might change, even though their normal bundles are all isomorphic to $(\mathcal{D}, J)$.

Let $\xi \in \kappa(\mathcal{D}, J)$. We may ask if there are canonical representatives of $\mathcal{S}(\xi, \bar{J})$. We can answer this question using a variational principle, as done in [13]. For let $\mathfrak{M}(\xi, \bar{J})$ be the set of all compatible Sasakian metrics arising from structures in $\mathcal{S}(\xi, \bar{J})$, and consider the functional

$$
\begin{aligned}
\mathfrak{M}(\xi, \bar{J}) & \rightarrow \mathbb{R} \\
g & \mapsto \int_{M} s_{g}^{2} d \mu_{g} .
\end{aligned}
$$

Its critical points define the canonical representatives that we seek. Furthermore, by the identification of $\kappa(\mathcal{D}, J)$ with $\mathfrak{t}_{k}^{+}$, we may then single out the set of Reeb vector fields $\xi$ in $\mathfrak{t}_{k}^{+}$for which the functional (10) admits critical points at all.

Definition 2.4. We say that $(\xi, \eta, \Phi, g) \in \mathcal{S}(\xi, \bar{J})$ is an extremal Sasakian structure if $g$ is a critical point of (10). A vector field $\xi \in \kappa(\mathcal{D}, J)$ is said to be extremal if there exists an extremal Sasakian structure in $\mathcal{S}(\xi, \bar{J})$. We denote by $\mathfrak{e}(\mathcal{D}, J)$ the set of all extremal elements of the Sasaki cone, and refer to it as the extremal Sasaki set of the CR structure $(\mathcal{D}, J)$.

Notice that $(\xi, \eta, \Phi, g) \in \mathcal{S}(\xi, \bar{J})$ is extremal if, and only if, the transversal metric $g^{T}$ is extremal in the Kähler sense [13. We also have that $\mathfrak{e}(\mathcal{D}, J)$ is an open subset of $\kappa(\mathcal{D}, J)$ [13, a Sasakian version of the the openness theorem in Kähler geometry [27, 38.

\section{The Energy functional in the SASAKI CONE}

By Lemma 2.3, given any Sasakian structure $(\xi, \eta, \Phi, g)$ in $\mathcal{S}(\mathcal{D}, J)$, the space of $g^{T}$-Killing potentials associated to a maximal torus has dimension $k-1$. We denote by $\mathcal{H}_{\xi}=\mathcal{H}_{\xi, g}(\mathcal{D}, J)$ the vector subspace of $C^{\infty}(M)$ that they and the constant functions span. This is the space of functions whose transverse gradient is holomorphic.

Given a Sasakian metric $g$ such that $(\xi, \eta, \Phi, g) \in \mathcal{S}(\mathcal{D}, J)$, we define the map

$$
\begin{array}{cl}
C^{\infty}(M) & \rightarrow \mathcal{H}_{\xi} \\
f & \mapsto \pi_{g} f
\end{array}
$$

to be the $L^{2}$-orthogonal projection with $\mathcal{H}_{\xi}$ as its range. Then the metric $g$ in $\mathfrak{M}(\xi, \bar{J})$ is extremal if, and only if, the scalar curvature $s_{g}=s_{g}^{T}-2 n$ is equal to its projection onto $\mathcal{H}_{\xi}$ [39. Or said differently, if, and only if, $s_{g}$ is an affine function of the space of Killing potentials. The functional (10) then admits the lower bound

$$
\mathfrak{M}(\xi, \bar{J}) \ni g \mapsto \int s_{g}^{2} d \mu_{g} \geq \int\left(\pi_{g} s_{g}\right)^{2} d \mu_{g},
$$


where the right side only depends upon the polarization $(\xi, \mathcal{D}, J)$, and it is only reached if the metric $g$ is extremal. This reproduces in this context a situation analyzed in the Kähler case already [40, 41].

We define the energy of a Reeb vector field in the Sasakian cone to be the functional given by this optimal lower bound:

$$
\begin{array}{cl}
\kappa(\mathcal{D}, J) & \stackrel{E}{\rightarrow} \mathbb{R} \\
\xi & \mapsto \int\left(\pi_{g} s_{g}\right)^{2} d \mu_{g},
\end{array}
$$

Definition 3.1. A Reeb vector field $\xi \in \kappa(\mathcal{D}, J)$ is said to be strongly extremal if it is a critical point of the functional (12) over the space of Sasakian structures in $\kappa(\mathcal{D}, J)$ that fix the volume of $M$. The Sasakian structure $(\xi, \eta, \Phi, g)$ is said to be a strongly extremal representative of $\kappa(\mathcal{D}, J)$ if $\xi$ is strongly extremal, and if $g \in \mathcal{S}(\xi, \bar{J})$ is an extremal representative of the polarized manifold $(M, \xi, J)$.

\section{The Euler-Lagrange Equation for strongly extremal Reeb Vector FIELDS}

We now discuss some of the variational results that play a rôle in the derivation of the Euler-Lagrange equation of a strongly extremal Reeb vector field. Complete details of this derivation will appear elsewhere.

Let us consider a path $\xi_{t} \in \kappa(\mathcal{D}, J)$, and a corresponding path of Sasakian structures $\left(\xi_{t}, \eta_{t}, \Phi_{t}, g_{t}\right)$. The path of contact forms must be of the type

$$
\eta_{t}=\frac{1}{\eta\left(\xi_{t}\right)} \eta+\frac{1}{2} d^{c} \varphi_{t}
$$

where $\varphi_{t}$ is a $\xi_{t}$-basic function. For convenience, we drop the subscript when we consider the value of quantities at $t=0$. Then, we have that $\eta(\xi)=1, \varphi$ is constant, and $\dot{\varphi}$ a $\xi$-basic function.

Proposition 4.1. Let $\left(\xi_{t}, \eta_{t}, \Phi_{t}, g_{t}\right)$ be a path of Sasakian structures that starts at $(\xi, \eta, \Phi, g)$, such that $\xi_{t} \in \kappa(\mathcal{D}, J)$ and $\eta_{t}$ is of the form (13). Then we have that

$$
d \mu_{t}=1-t\left((n+1) \eta(\dot{\xi})+\frac{1}{2} \Delta_{B} \dot{\varphi}\right) d \mu+O\left(t^{2}\right)
$$

and

$$
\frac{d}{d t} \mu_{g_{t}}(M)=-(n+1) \int(\eta(\dot{\xi})) d \mu .
$$

Volume preserving Sasakian deformations are given by variations $\xi_{t}$ of the Reeb vector field whose infinitesimal deformation is globally orthogonal to it, that is to say, such that $\eta(\dot{\xi})$ is orthogonal to the constants. Finally,

$$
D^{2} \mu(\dot{\xi}, \dot{\beta})=(n+1)(n+2) \int(\eta(\dot{\xi}))(\eta(\dot{\beta})) d \mu .
$$

Proof. By differentiation of (3), we obtain the variational expression for the volume form. The expression for the infinitesimal variation of the volume follows by Stokes' theorem, and the one for the Hessian by an iteration of this argument. 
Proposition 4.2. Let $\left(\xi_{t}, \eta_{t}, \Phi_{t}, g_{t}\right)$ be a path of Sasakian structures that starts at $(\xi, \eta, \Phi, g)$, such that $\xi_{t} \in \kappa(\mathcal{D}, J)$ and $\eta_{t}$ is of the form (13). Then we have the expansion

$$
s_{t}=s^{T}-2 n-t\left(n \Delta_{B}(\eta(\dot{\xi}))-s^{T} \eta(\dot{\xi})+\frac{1}{2} \Delta_{B}^{2} \dot{\varphi}+2\left(\rho^{T}, i \partial \bar{\partial} \dot{\varphi}\right)\right)+O\left(t^{2}\right),
$$

for the scalar curvature of $g_{t}$.

Proof. We have the relation $s_{t}=s_{t}^{T}-2 n$, where $s_{t}^{T}$ is the scalar curvature of the transverse Kähler metric $d \eta_{t}$. Since the latter is

$$
s_{t}^{T}=-2\left(d \eta_{t}, i \partial \bar{\partial} \log \operatorname{det}\left(d \eta_{t}\right)\right)_{d \eta_{t}},
$$

taking the $t$-derivative, and evaluating at $t=0$, we obtain the desired result. Just notice that the $d \eta$-trace of $d \dot{\eta}$ is equal to the $d \eta$-trace of $-\eta(\dot{\xi}) d \eta+i \partial \bar{\partial} \dot{\varphi}$, as the trace of $d(\eta(\dot{\xi})) \wedge \eta$ is zero.

We may now sketch the computation of the variation of the functional (12) along the curve $\xi_{t}$. If the subscript $t$ denotes quantities associated to the Sasakian structure $\left(\xi_{t}, \eta_{t}, \Phi_{t}, g_{t}\right)$, we have that

$$
\frac{d}{d t} E\left(\xi_{t}\right)=2 \int\left(\pi_{t} s_{t}\right)\left(\frac{d}{d t}\left(\pi_{t} s_{t}\right)\right) d \mu_{t}+\int\left(\pi_{t} s_{t}\right)^{2} \frac{d}{d t} d \mu_{t}
$$

and by the results of the previous propositions, the value at $t=0$ is given by

(14) $\left.\frac{d}{d t} E\left(\xi_{t}\right)\right|_{t=0}=2 \int(\pi s)\left[\left(-n \Delta_{B}+s^{T}\right) \eta(\dot{\xi})+\dot{\pi} s\right] d \mu-(n+1) \int(\pi s)^{2} \eta(\dot{\xi}) d \mu$.

We may compute the variation of $\pi$, but need less than that for our purposes. Indeed, if $G_{g}$ is the Green's operator of the metric $g$, we have that

$$
\left.\int(\pi s) \dot{\pi} s d \mu=\int\left(s^{T}-\pi s^{T}\right) 2 i G_{g} \bar{\partial}_{g}^{*}\left(\partial^{\#} \pi s\right\lrcorner d \dot{\eta}\right),
$$

where given a basic function $f, \partial^{\#} f$ is the operator obtained by raising the indices of the $(0,1)$ form $\bar{\partial} f$ using the transversal Kähler metric $d \eta$, and since

$$
\left.i \partial_{g}^{\#} \pi s\right\lrcorner d \dot{\eta}=-\bar{\partial}(\pi s \eta(\dot{\xi})),
$$

we obtain that

$$
\left.\int(\pi s) \dot{\pi} s d \mu=\int\left(s^{T}-\pi s^{T}\right) 2 i G_{g} \bar{\partial}_{g}^{*}\left(\partial^{\#} \pi s\right\lrcorner d \dot{\eta}\right)=-\int\left(s^{T}-\pi s^{T}\right) \pi s \eta(\dot{\xi}) d \mu,
$$

where the last equality follows because $s^{T}-\pi s^{T}$ is orthogonal to the constants.

Combining these results, we obtain the following:

Theorem 4.3. Let $\left(\xi_{t}, \eta_{t}, \Phi_{t}, g_{t}\right)$ be a path of volume preserving Sasakian structures that starts at $(\xi, \eta, \Phi, g)$, such that $\xi_{t} \in \kappa(\mathcal{D}, J)$, and $\eta_{t}$ is of the form (13) with $\varphi_{t}$ a constant. Then we have that

$$
\left.\frac{d}{d t} E\left(\xi_{t}\right)\right|_{t=0}=2 \int(\pi s)\left[\left(-n \Delta_{B}+\pi s^{T}\right) \eta(\dot{\xi})\right] d \mu-(n+1) \int(\pi s)^{2} \eta(\dot{\xi}) d \mu .
$$

The Reeb vector field $\xi \in \kappa(\mathcal{D}, J)$ is a critical point of (12) over the space of deformations with $g_{t}$ of fixed volume if, and only if,

$$
\pi_{g}\left[-2 n \Delta_{B} \pi_{g} s_{g}-(n-1)\left(\pi_{g} s_{g}\right)^{2}\right]+4 n \pi_{g} s_{g}=\lambda,
$$

for $\lambda$ a constant. 
4.1. Some examples of critical Reeb vector fields. We may define a character that obstructs the existence of extremal Sasakian structures $(\xi, \eta, \Phi, g) \in \mathcal{S}(\xi, \bar{J})$ where the scalar curvature $s_{g}$ of $g$ is constant [13. This is analogous to the Futaki invariant [16, 14] in the Kähler category.

Indeed, for any transversally holomorphic vector field $\partial_{g}^{\#} f$ with Killing potential $f$, the Sasaki-Futaki invariant is given by

$$
\mathfrak{F}_{\xi}\left(\partial_{g}^{\#} f\right)=-\int f\left(s_{g}-s_{0}\right) d \mu_{g},
$$

where $g$ is any Sasakian metric in $\mathfrak{M}(\xi, \bar{J}), s_{g}$ is its scalar curvature, $s_{0}$ is the projection of $s_{g}$ onto the constants. This expression defines uniquely a character on the entire algebra of transversally holomorphic vector fields, and if $(\xi, \eta, \Phi, g)$ is an extremal Sasakian structure in $\mathcal{S}(\xi, \bar{J})$, the scalar curvature $s_{g}$ is constant if, and only if, $\mathfrak{F}_{\xi} \equiv 0$.

Proposition 4.4. Suppose that $(\xi, \eta, \Phi, g) \in \mathcal{S}(\xi, \bar{J})$ is such that $\pi_{g} s_{g}$ is a constant. Then, for any other Sasakian structure $(\xi, \tilde{\eta}, \tilde{\Phi}, \tilde{g})$ in $\mathcal{S}(\xi, \bar{J})$, we have that $\pi_{\tilde{g}} s \tilde{g}$ is constant also, and equal to $\pi_{g} s_{g}$.

Proof. The proof follows by the same reasoning used to prove an analogous statement in the Kähler context (cf. [42, Proposition 5).

Theorem 4.5. Let $\xi \in \kappa(\mathcal{D}, J)$ be such that the Sasaki-Futaki invariant is identically zero, $\mathfrak{F}_{\xi}(\cdot)=0$. Then $\xi$ is a critical point of (12).

Proof. If $\mathfrak{F}_{\xi}(\cdot)=0$, then $\pi_{g} s_{g}$ is constant, and $\xi$ satisfies (17).

Example 4.6. Under the assumption that the cone $C(M)=M \times \mathbb{R}^{+}$is a CalabiYau manifold, the Hilbert action over Sasakian metrics has been extensively analyzed [29]. When considered as a function of Reeb vector fields of charge $n+1$, this

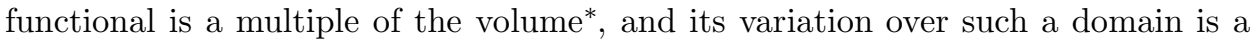
constant multiple of the Sasaki-Futaki invariant. Thus, a critical Reeb vector field $\xi$ has vanishing Sasaki-Futaki invariant, and if $(\xi, \eta, \Phi, g)$ is a Sasakian structure corresponding to it, the function $\pi_{g} s_{g}$ is necessarily constant. By the convexity of the volume functional (see Proposition 4.1), the critical Reeb vector field of this functional is unique. We thus see that the critical Reeb field singled out by the Hilbert action, on this type of special manifolds, must be a critical point of our functional (12) also, as was to be expected.

\section{SASAKIAN GEOMETRY OF LINKS OF ISOLATED HYPERSURFACE SINGULARITIES}

Many of the results we discuss from here on involve the natural Sasakian geometry occurring on links of isolated hypersurface singularities. In the spirit of self-containment, we provide a brief review of the geometry of links in arbitrary dimension, which we shall use mostly in dimension 5 later on.

Let us recall (cf. Chapters 4 and 9 of 7 ) that a polynomial $f \in \mathbb{C}\left[z_{0}, \ldots, z_{n}\right]$ is said to be a weighted homogeneous polynomial of degree $d$ and weight $\mathbf{w}=$ $\left(w_{0}, \ldots, w_{n}\right)$ if for any $\lambda \in \mathbb{C}^{\times}$

$$
f\left(\lambda^{w_{0}} z_{0}, \ldots, \lambda^{w_{n}} z_{n}\right)=\lambda^{d} f\left(z_{0}, \ldots, z_{n}\right) .
$$

\footnotetext{
*In earnest, this is done in [29 assuming that $M$ is quasi-regular, but the result holds in further generality. Notice also that in the statement for the charge of the Reeb vector field, we have made adjustments between the convention we use for the dimension of $M$, and the one in the said reference.
} 
We are interested in those weighted homogeneous polynomials $f$ whose zero locus in $\mathbb{C}^{n+1}$ has only an isolated singularity at the origin. We define the link $L_{f}(\mathbf{w}, d)$ as $f^{-1}(0) \cap \mathbb{S}^{2 n+1}$, where $\mathbb{S}^{2 n+1}$ is the $(2 n+1)$-sphere in Euclidean space. By the Milnor fibration theorem [30], $L_{f}(\mathbf{w}, d)$ is a closed $(n-2)$-connected manifold that bounds a parallelizable manifold with the homotopy type of a bouquet of $n$-spheres. Furthermore, $L_{f}(\mathbf{w}, d)$ admits a Sasaki-Seifert structure $\mathcal{S}=\left(\xi_{\mathbf{w}}, \eta_{\mathbf{w}}, \Phi_{\mathbf{w}}, g_{\mathbf{w}}\right)$ in a natural way [44, 5 . This structure is quasi-regular, and its bundle $(\mathcal{D}, J)$ has $c_{1}(\mathcal{D})=0$. This latter property implies that

$$
c_{1}\left(\mathcal{F}_{\xi_{\mathbf{w}}}\right)=a\left[d \eta_{\mathbf{w}}\right]_{B}
$$

for some constant $a$, where $\mathcal{F}_{\xi_{\mathrm{w}}}$ is the characteristic foliation. The sign of $a$ determines the negative, null, and positive cases that we shall refer to below.

The Sasaki-Futaki invariant (18) is the obstruction for the scalar curvature of the metric $g$ in an extremal Sasakian structure $\mathcal{S}=(\xi, \eta, \Phi, g)$ to be constant [13. One particular case where this obstruction vanishes is when there are no transversally holomorphic vector fields other than those generated by $\xi$ itself. In that case, the Sasaki cone of the corresponding CR structure turns out to be of dimension one. If the Sasakian structure is quasi-regular, the transversally holomorphic vector fields are the lifts of holomorphic vector fields on the projective algebraic variety $z=M / \mathbb{S}_{\xi}^{1}$, where $\mathbb{S}_{\xi}^{1}$ is the circle generated by the Reeb vector field.

Let us recall that a Sasakian metric $g$ is said to be $\eta$-Einstein if

$$
\operatorname{Ric}_{g}=\lambda g+\nu \eta \otimes \eta
$$

for some constants $\lambda$ and $\nu$ that satisfy the relation $\lambda+\nu=2 n$. They yield particular examples of Sasakian metrics of constant scalar curvature, a function which in these cases is given in terms of $\lambda$ by the expression $s_{g}=2 n(1+\lambda)$.

The only transversally holomorphic vector fields of a negative or a null link of an isolated hypersurface singularity are those generated by the Reeb vector field. These links have a one-dimensional Sasaki cone, and by the transverse Aubin-Yau theorem [11, any point in this cone can be represented by a Sasakian structure whose metric is $\eta$-Einstein (see examples of such links in [8, 7]). We thus obtain the following

Theorem 5.1. Let $L_{f}(\mathbf{w}, d)$ be either a negative or null link of an isolated hypersurface singularity with underlying $\mathrm{CR}$ structure $(\mathcal{D}, J)$. Then its Sasaki cone $\kappa(\mathcal{D}, J)$ is one-dimensional, and it coincides with the extremal Sasaki set $\mathfrak{e}(\mathcal{D}, J)$.

The case of positive links is more complicated. This can be deduced already from the fact that if we were to have a Sasakian structure $\mathcal{S}=(\xi, \eta, \Phi, g)$ such that $c_{1}\left(\mathcal{F}_{\xi}\right)=a[d \eta]_{B}$ with $a>0$ and where $g$ is $\eta$-Einstein, then there would exist a transverse homothety (9) that would yield a Sasaki-Einstein metric of positive scalar curvature, and generally speaking, these types of metrics are difficult to be had.

In order to understand this case better, we begin by stating the following result that applies to links in general, and which follows by Corollary 5.3 of [13:

Proposition 5.2. Let $L_{f}(\mathbf{w}, d)$ be the link of an isolated hypersurface singularity with its natural Sasakian structure $\mathcal{S}=\left(\xi_{\mathbf{w}}, \eta_{\mathbf{w}}, \Phi_{\mathbf{w}}, g_{\mathbf{w}}\right)$. If $g \in \mathcal{S}\left(\xi_{\mathbf{w}}, \bar{J}\right)$ is an extremal Sasakian metric with constant scalar curvature, then $g$ is $\eta$-Einstein. 
By Lemma 5.5.3 of [7, if the weight vector $\mathbf{w}=\left(w_{0}, \ldots, w_{n}\right)$ of a link $L_{f}(\mathbf{w}, d)$ is such that $2 w_{i}<d$ for all but at most one of the indices, then the Lie algebra $\mathfrak{a u t}(\mathcal{S})$ is generated by the Reeb vector field $\xi_{\mathrm{w}}$. We have the following

Theorem 5.3. Let $L_{f}(\mathbf{w}, d)$ be the link of an isolated hypersurface singularity with $2 w_{i}<d$ for all but at most one of the indices, and let $(\mathcal{D}, J)$ by its underlying $\mathrm{CR}$ structure. Then the dimension of the Sasaki cone $\kappa(\mathcal{D}, J)$ is one.

Even if we assume that for a given positive link $L_{f}(\mathbf{w}, d)$ the hypothesis of Theorem 5.3 apply, and so its Sasaki-Futaki invariant vanishes identically, there could be other obstructions preventing the existence of extremal Sasakian metrics of constant scalar curvature on it. Indeed, as was observed in [19], classical estimates of Bishop and Lichnerowicz may obstruct the existence of Sasaki-Einstein metrics, an observation that has produced a very effective tool to rule out the existence of these metrics in various cases. Employing the Lichnerowicz estimates [19] in combination with the discussion above and some results in [13, we obtain the following:

Proposition 5.4. Let $L_{f}(\mathbf{w}, d)$ be a link of an isolated hypersurface singularity with its natural Sasakian structure, and let $I:=|\mathbf{w}|-d=\left(\sum_{j} w_{j}\right)-d$ be its index. If

$$
I>n \min _{i}\left\{w_{i}\right\},
$$

then $L_{f}(\mathbf{w} ; d)$ cannot admit any Sasaki-Einstein metric. In that case, $\mathcal{S}\left(\xi_{\mathbf{w}}, \bar{J}\right)$ does not admit extremal representatives.

Combining Theorem 5.3 and Proposition 5.4 we may obtain examples of positive links whose Sasaki cones are one dimensional, and whose extremal sets are empty.

Theorem 5.5. Let $L_{f}(\mathbf{w}, d)$ be a positive link of an isolated hypersurface singularity whose index I satisfies the relation $I>n \min _{i}\left\{w_{i}\right\}$, and such that $2 w_{i}<d$ for all but at most one of the indices. If $(\mathcal{D}, J)$ is its underlying $\mathrm{CR}$ structure, we have that the dimension of the Sasaki cone $\kappa(\mathcal{D}, J)$ is one, and that the extremal set $\mathfrak{e}(\mathcal{D}, J)$ is empty.

Besides the conditions given above on the weight vector of a link, there are other interesting algebraic conditions that suffice to prove the existence of extremal Sasakian metrics on it. These arise when we view the link as a Seifert fibration over a base $X$ with orbifold singularities. If the Kähler class of the projective algebraic variety $X$ is a primitive integral class in the second orbifold cohomology group, and the constant $a$ in (19) is positive, they serve to measure the singularity of the pair $\left(X, K^{-1}+\Delta\right)$, where $K^{-1}$ is an anti-canonical divisor, and $\Delta$ is a branch divisor, and are known as Kawamata log terminal (Klt) conditions (cf. Chapter 5 of [7]).

A weighted homogeneous polynomial of the form

$$
f=z_{0}^{a_{0}}+\cdots+z_{n}^{a_{n}}, \quad a_{i} \geq 2,
$$

is called a Brieskorn-Pham (BP) polynomial. In this case the exponents $a_{i}$, the weights $w_{i}$, and degree are related by $d=a_{i} w_{i}$ for each $i=0, \ldots, n$. We change slightly the notation in this case, and denote by $L_{f}(\mathbf{a}), \mathbf{a}=\left(a_{0}, \ldots, a_{n}\right)$, the link that a BP polynomial $f$ defines. These are special but quite important examples of links. Their Klt conditions were described in [9]. The base of a BP link $L_{f}(\mathbf{a})$ 
admits a positive Kähler-Einstein orbifold metric if

$$
1<\sum_{i=0}^{n} \frac{1}{a_{i}}<1+\frac{n}{n-1} \min _{i, j}\left\{\frac{1}{a_{i}}, \frac{1}{b_{i} b_{j}}\right\} .
$$

where $b_{j}=\operatorname{gcd}\left(a_{j}, C^{j}\right)$ and $C^{j}=\operatorname{lcm}\left\{a_{i}: i \neq j\right\}$. This condition leads to the finding of a a rather large number of examples of Sasaki-Einstein metrics on homotopy spheres [9, 10] and rational homology spheres [4, 25].

In the special case when the integers $\left(a_{0}, \ldots, a_{n}\right)$ are pairwise relatively prime, Ghigi and Kollár [20] obtained a sharp estimate. In this case, the BP link is always a homotopy sphere, and if we combine the now sharp Klt estimate with Proposition 5.4 above, we see that, when the $a_{i}$ s are pairwise relatively prime, a BP link $L_{f}(\mathbf{a})$ admits an extremal Sasakian metric if, and only if,

$$
\sum_{i=0}^{n} \frac{1}{a_{i}}<1+n \min _{i}\left\{\frac{1}{a_{i}}\right\} .
$$

Other applications of the Klt estimate (21) exist [8, 7]. If $f$ is neither a BP polynomial nor a perturbation thereof, alternative estimates must be developed. Further details can be found in $[7$.

Notice that in all the link examples we have discussed above, information about the topology of the underlying manifold is absent. We end this section addressing that issue in part.

Topological information about links was first given by Milnor and Orlik in [31] through the study of the Alexander polynomial. They computed the Betti numbers of the manifold underlying the link of any weighted homogeneous polynomial of an isolated hypersurface singularity, and in the case of a rational homology sphere, the order of the relevant homology groups. A bit later, Orlik 32 postulated a combinatorial conjecture for computing the torsion, an algorithm that we describe next (see Chapter 9 of 7 for more detail).

Given a link $L_{f}(\mathbf{w}, d)$, we define its fractional weights to be

$$
\left(\frac{d}{w_{0}}, \cdots, \frac{d}{w_{n}}\right) \equiv\left(\frac{u_{0}}{v_{0}}, \cdots, \frac{u_{n}}{v_{n}}\right)
$$

where

$$
u_{i}=\frac{d}{\operatorname{gcd}\left(d, w_{i}\right)}, \quad v_{i}=\frac{w_{i}}{\operatorname{gcd}\left(d, w_{i}\right)} .
$$

We denote by $(\mathbf{u}, \mathbf{v})$ the tuple $\left(u_{0}, \ldots, u_{n}, v_{0}, \ldots, v_{n}\right)$. By (23), we may go between $(\mathbf{w}, d)$ and $(\mathbf{u}, \mathbf{v})$. We will sometimes write $L_{f}(\mathbf{u}, \mathbf{v})$ for $L_{f}(\mathbf{w}, d)$.

Definition 5.6. (Orlik's algorithm) Let $\left\{i_{1}, \ldots, i_{s}\right\} \subset\{0,1, \ldots, n\}$ be an ordered set of $s$ indices, that is to say, $i_{1}<i_{2}<\cdots<i_{s}$. Let us denote by $I$ its power set (consisting of all of the $2^{s}$ subsets of the set), and by $J$ the set of all proper subsets. Given a $(2 n+2)$-tuple $(\mathbf{u}, \mathbf{v})=\left(u_{0}, \ldots, u_{n}, v_{0}, \ldots, v_{n}\right)$ of integers, we define inductively a set of $2^{s}$ positive integers, one for each ordered element of $I$, as follows:

$$
c_{\emptyset}=\operatorname{gcd}\left(u_{0}, \ldots, u_{n}\right),
$$

and if $\left\{i_{1}, \ldots, i_{s}\right\} \in I$ is ordered, then

$$
c_{i_{1}, \ldots, i_{s}}=\frac{\operatorname{gcd}\left(u_{0}, \ldots, \hat{u}_{i_{1}}, \ldots, \hat{u}_{i_{s}}, \ldots, u_{n}\right)}{\prod_{J} c_{j_{1}, \ldots j_{t}}} .
$$


Similarly, we also define a set of $2^{s}$ real numbers by

$$
k_{\emptyset}=\epsilon_{n+1},
$$

and

$$
k_{i_{1}, \ldots, i_{s}}=\epsilon_{n-s+1} \sum_{J}(-1)^{s-t} \frac{u_{j_{1}} \cdots u_{j_{t}}}{v_{j_{1}} \cdots v_{j_{t}} \operatorname{lcm}\left(u_{j_{1}}, \ldots, u_{j_{t}}\right)},
$$

where

$$
\epsilon_{n-s+1}= \begin{cases}0 & \text { if } n-s+1 \text { is even, } \\ 1 & \text { if } n-s+1 \text { is odd, }\end{cases}
$$

respectively. Finally, for any $j$ such that $1 \leq j \leq r=\left[\max \left\{k_{i_{1}, \ldots, i_{s}}\right\}\right]$, where $[x]$ is the greatest integer less or equal than $x$, we set

$$
d_{j}=\prod_{k_{i_{1}}, \ldots, i_{s} \geq j} c_{i_{1}, \ldots, i_{s}} .
$$

Orlik's torsion conjecture [32] is stated in terms of the integers computed by this algorithm:

Conjecture 5.7. For a link $L_{f}(\mathbf{u}, \mathbf{v})$ with fractional weights $(\mathbf{u}, \mathbf{v})$, we have

$$
H_{n-1}\left(L_{f}(\mathbf{u}, \mathbf{v}), \mathbb{Z}\right)_{\text {tor }}=\mathbb{Z} / d_{1} \oplus \cdots \oplus \mathbb{Z} / d_{r} .
$$

This conjecture is known to hold in certain special cases [36, 34].

Proposition 5.8. Conjecture 5.7 holds in the following cases:

(1) In dimension 3 , that is to say, when $n=2$.

(2) For Brieskorn-Pham polynomials (20).

(3) For $f(\mathbf{z})=z_{0}^{a_{0}}+z_{0} z_{1}^{a_{1}}+z_{1} z_{2}^{a_{2}}+\cdots+z_{n-1} z_{n}^{a_{n}}$.

It is also known to hold for certain complete intersections given by generalized Brieskorn polynomials [36].

Before his tragic accident, by using Kollár's Theorem 6.1 below, Kris proved that Conjecture 5.7 holds in dimension 5 also. We present his argument in the appendix.

\section{Extremal Sasakian metrics in Dimension five}

We start by reviewing briefly some general facts about Sasakian geometry on simply connected 5-manifolds (cf. Chapter 10 of [7]).

6.1. Sasakian geometry in dimension five. Sasakian geometry in dimension five is large enough to be interesting while remaining manageable, at least in the case of closed simply connected manifolds where we can use the Smale-Barden classification.

Up to diffeomorphism, Smale 43 classified all closed simply connected 5-manifolds that admit a spin structure, and showed they must be of the form

$$
M=k M_{\infty} \# M_{m_{1}} \# \cdots \# M_{m_{n}}
$$

where $M_{\infty}=\mathbb{S}^{2} \times \mathbb{S}^{3}, k M_{\infty}$ is the $k$-fold connected sum of $M_{\infty}, m_{i}$ is a positive integer with $m_{i}$ dividing $m_{i+1}$ and $m_{1} \geq 1$, and where $M_{m}$ is $\mathbb{S}^{5}$ if $m=1$, or a 5-manifold such that $H_{2}\left(M_{m}, \mathbb{Z}\right)=\mathbb{Z} / m \oplus \mathbb{Z} / m$, otherwise. The integer $k$ in this expression can take on the values $0,1, \ldots$, with $k=0$ corresponding to the case where there is no $M_{\infty}$ factor at all. It will be convenient to set the convention 
$0 M_{\infty}=\mathbb{S}^{5}$ below, which is consistent with the fact that the sphere is the neutral element for the connected sum operation. The $m_{i}$ s can range in $1,2, \ldots$.

Barden [2] extended Smale's classification to include the non-spin case also, where we must add the non-trivial $\mathbb{S}^{3}$-bundle over $\mathbb{S}^{2}$, denoted by $X_{\infty}$, and certain rational homology spheres $X_{j}$ parametrized by $j=-1,1,2, \ldots$ that do not admit contact structures, and can be safely ignored from consideration when studying the Sasakian case. Notice that $X_{\infty} \# X_{\infty}=2 M_{\infty}$, so in considering simply connected Sasakian 5 -manifolds that are non-spin, it suffices to take a connected sum of the manifold (27) with one copy of $X_{\infty}$, or equivalently, replace at most one copy of $M_{\infty}$ with $X_{\infty}$.

What Smale-Barden manifolds can admit Sasakian structures? A partial answer to this question has been given recently by Kollár [24. In order to present this, it is convenient to describe the torsion subgroup of $H_{2}(M, \mathbb{Z})$ for the manifold $M$ in terms of elementary divisors rather than the invariant factors in (27). The group $H_{2}(M, \mathbb{Z})$ can be written as a direct sum of cyclic groups of prime power order

$$
H_{2}(M, \mathbb{Z})=\mathbb{Z}^{k} \oplus \bigoplus_{p, i}\left(\mathbb{Z} / p^{i}\right)^{c\left(p^{i}\right)},
$$

where $k=b_{2}(M)$ and $c\left(p^{i}\right)=c\left(p^{i}, M\right)$. These non-negative integers are determined by $H_{2}(M, \mathbb{Z})$, but the subgroups $\mathbb{Z} / p^{i} \subset H_{2}(M, \mathbb{Z})$ are not unique. We can choose the decomposition (28) such that the second Stiefel-Whitney class map

$$
w_{2}: H_{2}(M, \mathbb{Z}) \rightarrow \mathbb{Z} / 2
$$

is zero on all but one of the summands $\mathbb{Z} / 2^{j}$. If we now assume that $M$ carries a Sasakian structure, by Rukimbira's approximation theorem [37, $M$ admits a Seifert fibered structure (in general, non-unique) with an associated Sasakian structure, or a Sasaki-Seifert structure as referred to in [7. Kollár 24] proved that the existence of a Seifert fibered structure on $M$ imposes constraints on the invariants $k$ and $c\left(p^{i}\right)$ of (28) above. Namely, for the primes that appear in the expression, the cardinality of the set $\left\{i: c\left(p^{i}\right)>0\right\}$ must be less or equal than $k+1$. For example, both $M_{2}$ and $M_{4}$ admit Sasakian structures but $M_{2} \# M_{4}$ does not. Other obstructions involving the torsion subgroup of $H_{2}(M, \mathbb{Z})$ apply as well $[24,7]$.

The case of positive Sasakian structures is particularly attractive since they provide examples of Riemannian metrics with positive Ricci curvature. Kollár [25] (see also [26] in these Proceedings) has shown that positivity greatly restricts the allowable torsion groups. He proved that if a closed simply connected 5-manifold $M$ admits a positive Sasakian structure, then the torsion subgroup of $H_{2}(M, \mathbb{Z})$ must be one of the following:

(1) $(\mathbb{Z} / m)^{2}, m \in \mathbb{Z}^{+}$,

(2) $(\mathbb{Z} / 5)^{4}$

(3) $(\mathbb{Z} / 4)^{4}$,

(4) $(\mathbb{Z} / 3)^{4},(\mathbb{Z} / 3)^{6}$, or $(\mathbb{Z} / 3)^{8}$,

(5) $(\mathbb{Z} / 2)^{2 n}, n \in \mathbb{Z}^{+}$.

Conversely, for each finite group $G$ in the list above, there is a closed simply connected 5-manifold $M$ with $H_{2}(M, \mathbb{Z})_{\text {tor }}=G$, and remarkably enough, all of these manifolds can be realized as the links of isolated hypersurface singularities.

Let us recall that a quasi-regular Sasakian structure can be viewed as a Seifert fibered structure. In dimension five, Kollár 25] has shown how the branch divisors 
$D_{i}$ of the orbifold base determine the torsion in $H_{2}(M, \mathbb{Z})$. For simply connected 5 -manifolds, this result is as follows:

Theorem 6.1. Let $M^{5}$ be a compact simply connected 5-manifold with a quasiregular Sasakian structure $\mathcal{S}$, let $\left(\mathcal{Z}, \sum_{i}\left(1-\frac{1}{m_{i}}\right) D_{i}\right)$ denote the corresponding projective algebraic orbifold base, with branch divisors $D_{i}$ and ramification index $m_{i}$, and let $k$ be the second Betti number of $M^{5}$. Then the integral cohomology groups of $M^{5}$ are as follows:

\begin{tabular}{|c|c|c|c|c|c|c|}
\hline \hline$i$ & 0 & 1 & 2 & 3 & 4 & 5 \\
\hline \hline$H^{i}\left(M^{5}, \mathbb{Z}\right)$ & $\mathbb{Z}$ & 0 & $\mathbb{Z}^{k}$ & $\mathbb{Z}^{k} \oplus \sum_{i}\left(\mathbb{Z} / m_{i}\right)^{2 g\left(D_{i}\right)}$ & 0 & $\mathbb{Z}$ \\
\hline
\end{tabular}

where $g(D)$ is the genus of the Riemann surface $D$ (notice that $\mathbb{Z} / 1$ is the trivial group).

6.2. Existence of Extremal Metrics. Here we reproduce a table [7] that lists all simply connected spin 5-manifolds that can admit a Sasaki-Einstein metric (and which are, therefore, Sasaki extremal). In the first column we list the type of manifold in terms of Smale's description (27), while in the second column we indicate restrictions on these under which a Sasaki-Einstein structure is known to exist. Any Smale manifold that is not listed here cannot admit a Sasaki-Einstein metric, but could, in principle, admit an extremal metric. We list the manifolds but not the number of deformation classes of positive Sasakian structures that may occur, a number that varies depending upon the manifold in question. For example, there are infinitely many such deformation classes on the 5-sphere $\mathbb{S}^{5}$ and on $k\left(\mathbb{S}^{2} \times \mathbb{S}^{3}\right)$, as well as on some rational homology spheres. On the other hand, there is a unique deformation class of positive Sasakian structures on the rational homology spheres $2 M_{5}$ and $4 M_{3}$ [25, 26], and they both admit extremal metrics.

\begin{tabular}{|c|c|}
\hline \hline Manifold $M$ & S-E \\
\hline \hline$k M_{\infty}, k \geq 0$ & any $k$ \\
\hline $8 M_{\infty} \# M_{m}, m>2$ & $m>4$ \\
\hline $7 M_{\infty} \# M_{m}, m>2$ & $m>2$ \\
\hline $6 M_{\infty} \# M_{m}, m>2$ & $m>2$ \\
\hline $5 M_{\infty} \# M_{m}, m>2$ & $m>11$ \\
\hline $4 M_{\infty} \# M_{m}, m>2$ & $m>4$ \\
\hline $3 M_{\infty} \# M_{m}, m>2$ & $m=7,9$ or $m>10$ \\
\hline $2 M_{\infty} \# M_{m}, m>2$ & $m>11$ \\
\hline$M_{\infty} \# M_{m}, m>2$ & $m>11$ \\
\hline$M_{m}, m>2$ & $m>2$ \\
\hline $2 M_{5}, 2 M_{4}, 4 M_{3}, M_{\infty} \# 2 M_{4}$ & yes \\
\hline$k M_{\infty} \# 2 M_{3}$ & $k=0$ \\
\hline$k M_{\infty} \# 3 M_{3}, k \geq 0$ & $k=0$ \\
\hline$k M_{\infty} \# n M_{2}, k \geq 0, n>0$ & $(k, n)=(0,1)$ or $(1, n), n>0$ \\
\hline$k M_{\infty} \# M_{m}, k>8,2<m<12$ & \\
\hline
\end{tabular}

Table 1. Simply connected spin 5-manifolds admitting Sasaki-Einstein metrics. The right column indicates the restriction that ensures that the manifold on the left carries a Sasaki-Einstein metric. 
6.3. Brieskorn-Pham links with no extremal Sasakian metrics. We now provide a table with examples of Brieskorn-Pham links $L_{f}(\mathbf{a})$ whose extremal sets are all empty. These are obtained by using Proposition 5.4 the required estimate on the index being easily checked. We list the link together with the range for its parameters, the underlying manifold, and the dimension of the Sasaki cone $\kappa$ of the corresponding CR structure. There are infinitely many such links (or Sasaki-Seifert structures) on each of the manifolds listed.

\begin{tabular}{|c|c|c|}
\hline \hline Manifold $M$ & Link $L_{f}(\mathbf{a})$ & $\operatorname{Dim} \kappa$ \\
\hline \hline$M_{1}$ & $L(2,3,3,6 l+1), l \geq 2$ & 1 \\
\hline$M_{1}$ & $L(2,3,3,6 l+5), l \geq 2$ & 1 \\
\hline$M_{1}$ & $L(2,2,2,2 l+1), l \geq 2$ & 2 \\
\hline$M_{\infty}$ & $L(2,2,2,2 l), l \geq 3$ & 2 \\
\hline $2 M_{\infty}$ & $L(2,3,3,2(3 l+1)), l \geq 2$ & 1 \\
\hline $2 M_{\infty}$ & $L(2,3,3,2(3 l+2)), l \geq 2$ & 1 \\
\hline $4 M_{\infty}$ & $L(2,3,3,6 l), l \geq 3$ & 1 \\
\hline $6 M_{\infty}$ & $L(2,3,4,12 l), l \geq 3$ & 1 \\
\hline $8 M_{\infty}$ & $L(2,3,5,30 l), l \geq 3$ & 1 \\
\hline$(k-1) M_{\infty}$ & $L(2,2, k, 2 l k), l \geq 2, k \geq 3$ & 2 \\
\hline $4 M_{2}$ & $L(2,3,5,15(2 l+1)), l \geq 2$ & 1 \\
\hline $2 M_{\infty} \# M_{2}$ & $L(2,3,4,6(2 l+1)), l \geq 2$ & 1 \\
\hline
\end{tabular}

Table 2. Some examples of 5-dimensional Brieskorn-Pham links whose associated space of Sasakian structures, $\mathcal{S}(\xi)$, does not have extremal Sasakian metrics. The last column lists the dimension of their Sasaki cones.

More examples can be had by considering general weighted homogeneous polynomials $f$ instead. In those cases, we go back to the notation $L_{f}(\mathbf{w}, d)$ for the link, w its weight vector, and $d$ its degree. Their systematic study should be possible by using the classification of normal forms of Yau and $\mathrm{Yu}$ [46. Here we content ourselves by giving a few examples. In all of these cases, the Sasaki cone is one dimensional.

\begin{tabular}{|c|c|c|}
\hline \hline Manifold $M$ & weight vector w & $d$ \\
\hline \hline $3 M_{\infty} \# M_{2}$ & $(2,2(4 l+3), 6 l+5,2(6 l+5)), l \geq 2$ & $4(6 l+5)$ \\
\hline $7 M_{\infty}$ & $(1,4 l+1,3 l+1,2(3 l+1)), l \geq 2$ & $4(3 l+1)$ \\
\hline$M_{4}$ & $(4,3(2 l+1), 4(2 l+1), 4(3 l+1)), l \geq 5$ & $12(2 l+1)$ \\
\hline
\end{tabular}

Table 3. 5-dimensional links of isolated hypersurface singularities that do not carry Sasakian extremal structure.

In the first row of this table the existence of positive Sasakian structures on the Smale-Barden manifold $3 M_{\infty} \# M_{2}$ is reported here for the first time. As indicated in the table, for $l \geq 2$ these links do not carry extremal Sasakian metrics. It is unknown if an extremal Sasakian metric exists when $l=1$, and generally speaking, it is not known whether an extremal Sasakian metric exists at all on $3 M_{\infty} \# M_{2}$. A similar situation occurs for the link $L_{f}((4,5,12,20), 40)$, which gives a positive Sasakian structure on the manifold $3 M_{\infty} \# M_{4}$, and which is not yet known to carry extremal Sasakian metrics. Thus, it is natural to pose the following: 
Question 6.1. Are there positive Sasakian manifolds that admit no extremal Sasakian metrics?

6.4. Toric Sasakian 5-manifolds. The study of contact toric $(2 n+1)$-manifolds goes back to [1, where it was shown that toric contact structures split into two types, those where the torus action is free, and those where it is not, with the free action case easily described, and the non-free case described via a Delzant type theorem when $n \geq 2$. A somewhat refined classification was obtained later [28, and the non-free case (again, for $n \geq 2$ ) was characterized in 3 by the condition that the Reeb vector field belongs to the Lie algebra of the $(n+1)$-torus acting on the manifold. These toric actions were called actions of Reeb type, and it was proved that every toric contact structure of Reeb type admits a compatible Sasakian structure. Here we content ourselves with describing the Sasaki cone for a simple but interesting example. For more on toric Sasakian geometry, we refer the reader to $12,17,15$.

Example 6.2. The Wang-Ziller manifold $M_{k_{1}, k_{2}}^{p_{1}, p_{2}}$ [45] is defined to be the total space of the $\mathbb{S}^{1}$-bundle over $\mathbb{C P}^{p_{1}} \times \mathbb{C P}^{p_{2}}$ whose first Chern class is $k_{1} \alpha_{1}+k_{2} \alpha_{2}, \alpha_{i}$ being the positive generator of $H^{2}\left(\mathbb{C P}^{p_{i}}, \mathbb{Z}\right)$ with $k_{1}, k_{2} \in \mathbb{Z}^{+}$. These manifolds all admit homogeneous Einstein metrics [45], and also admit homogeneous Sasakian structures [6]. However, they are Sasaki-Einstein only if $k_{1} \alpha_{1}+k_{2} \alpha_{2}$ is proportional to the first Chern class of $\mathbb{C P}^{p_{1}} \times \mathbb{C P}^{p_{2}}$, that is to say, only when $k_{1} \alpha_{1}+k_{2} \alpha_{2}$ and $\left(p_{1}+1\right) \alpha_{1}+\left(p_{2}+1\right) \alpha_{2}$ are proportional.

We are interested in $M_{k, l}^{p, q}$ as a toric Sasakian manifold, and treat the five dimensional case $M_{k_{1}, k_{2}}^{1,1}$ only. We shall also assume that $\operatorname{gcd}\left(k_{1}, k_{2}\right)=1$, for then $M_{k_{1}, k_{2}}^{1,1}$ is simply connected, and diffeomorphic to $\mathbb{S}^{2} \times \mathbb{S}^{3}$. If $k_{1}$ and $k_{2}$ are not relatively prime, the analysis of $M_{k_{1}, k_{2}}^{1,1}$ can be obtained easily from the simply connected case by taking an appropriate cyclic quotient. We shall also assume that if $\left(k_{1}, k_{2}\right) \neq(1,1)$, then $k_{1}<k_{2}$, for otherwise we can simply interchange the two $\mathbb{C P}^{1} \mathrm{~s}$.

We wish to find the Sasaki cone for $M_{k_{1}, k_{2}}^{1,1}$. For that, we identify this manifold with the quotient $\mathbb{S}^{3} \times \mathbb{S}^{3} / \mathbb{S}^{1}\left(k_{1}, k_{2}\right)$, where the $\mathbb{S}^{1}$ action on $\mathbb{S}^{3} \times \mathbb{S}^{3}$ is given by

$$
(\mathbf{x}, \mathbf{y}) \mapsto\left(e^{i k_{2} \theta} \mathbf{x}, e^{-i k_{1} \theta} \mathbf{y}\right),
$$

and points in $\mathbb{S}^{3}$ are viewed as points in $\mathbb{C}^{2}$. Alternatively, $M_{k_{1}, k_{2}}^{1,1}$ can be obtained by a Sasakian reduction [21] of the standard Sasakian structure $\mathcal{S}_{0}$ on $\mathbb{S}^{7}$ by the $\mathbb{S}^{1}\left(k_{1}, k_{2}\right)$ action, the moment map $\mu: \mathbb{S}^{7} \rightarrow \mathbb{R}$ being given by

$$
\mu=k_{2}\left(\left|z_{0}\right|^{2}+\left|z_{1}\right|^{2}\right)-k_{1}\left(\left|z_{2}\right|^{2}+\left|z_{3}\right|^{2}\right) .
$$

The zero level set is the product $\mathbb{S}^{3}\left(R_{1}\right) \times \mathbb{S}^{3}\left(R_{2}\right)$ of spheres whose radii are given by

$$
R_{i}^{2}=\frac{k_{i}}{k_{1}+k_{2}}
$$

The Sasakian structure on $M_{k_{1}, k_{2}}^{1,1}$ induced by this reduction is denoted by $\mathcal{S}_{k_{1}, k_{2}}=$ $(\xi, \eta, \Phi, g)$, where for ease of notation, we refrain from writing the tensor fields with the subscripts also. 
Let us consider coordinates $\left(z_{0}, z_{1}, z_{2}, z_{3}\right)$ in $\mathbb{C}^{4}$, where $z_{j}=x_{j}+i y_{j}$. Then the vector fields

$$
H_{i}=y_{i} \frac{\partial}{\partial x_{i}}-x_{i} \frac{\partial}{\partial y_{i}}, 0 \leq i \leq 3,
$$

restrict to vector fields on $\mathbb{S}^{7}$ that span the Lie algebra $\mathfrak{t}_{4}$ of the maximal torus $T^{4} \subset \mathbb{S O}(8)$. The base space $\mathbb{C P}^{1} \times \mathbb{C P}^{1}$ of $M_{k_{1}, k_{2}}^{1,1}$ is obtained from the zero level set $\mathbb{S}^{3}\left(R_{1}\right) \times \mathbb{S}^{3}\left(R_{2}\right)$ as the quotient by the free $T^{2}$-action given by $(\mathbf{x}, \mathbf{y}) \mapsto$ $\left(e^{i \theta_{1}} \mathbf{x}, e^{i \theta_{2}} \mathbf{y}\right)$. Let $\tilde{X}$ denote the vector field on $\mathbb{C P}^{1}$ generating the $\mathbb{S}^{1}$-action defined in homogeneous coordinates by $\left[\zeta_{0}, \zeta_{1}\right] \mapsto\left[e^{i \phi} \zeta_{0}, e^{-i \phi} \zeta_{1}\right]$, and denote by $\tilde{X}_{1}$ and $\tilde{X}_{2}$ the vector field $\tilde{X}$ on the two copies of $\mathbb{C P}^{1}$. We let $X_{1}$ and $X_{2}$ denote the lifts of these vector fields to $\mathfrak{a u t}\left(\mathcal{S}_{k_{1}, k_{2}}\right)$.

The Reeb vector field $\xi$ of $\mathcal{S}_{k_{1}, k_{2}}$ is that induced by the restriction of $H_{0}+H_{1}+$ $H_{2}+H_{3}$ to the zero level set $\mathbb{S}^{3}\left(R_{1}\right) \times \mathbb{S}^{3}\left(R_{2}\right)$ of the moment map $\mu$ (see Theorems 8.5.2 and 8.5.3 in [7]). The Lie algebra $\mathfrak{t}_{3}\left(k_{1}, k_{2}\right)$ of the 3 -torus of $\mathcal{S}_{k_{1}, k_{2}}$ is spanned by the Reeb vector field $\xi$, and the two vector fields $X_{1}$ and $X_{2}$ described above.

We have:

Lemma 6.3. The Sasaki cone $\kappa\left(\mathcal{D}_{k_{1}, k_{2}}, J\right)$ for the Wang-Ziller manifold $M_{k_{1}, k_{2}}^{1,1}$ is given by the set of all vector fields

$$
Z_{l_{0}, l_{1}, l_{2}}=l_{0} \xi+l_{1} X_{1}+l_{2} X_{2},\left(l_{0}, l_{1}, l_{2}\right) \in \mathbb{R}^{3},
$$

subject to the conditions

$$
l_{0}>0, \quad\left|k_{1} l_{1}+k_{2} l_{2}\right|<\left(k_{1}+k_{2}\right) l_{0} .
$$

Proof. The Sasaki cone $\kappa\left(\mathcal{D}_{k_{1}, k_{2}}, J\right)$ is equal to the set of $\left\{Z \in \mathfrak{t}_{3} \mid \eta(Z)>0\right\}$ of positive vector fields (see Theorem 2.2). The vector fields $X_{1}$ and $X_{2}$ are induced on the quotient $M_{k_{1}, k_{2}}^{1,1} \approx\left(\mathbb{S}^{3}\left(R_{1}\right) \times \mathbb{S}^{3}\left(R_{2}\right)\right) / \mathbb{S}^{1}\left(k_{1}, k_{2}\right)$ by the restrictions of $H_{0}-H_{1}$ and $H_{2}-H_{3}$ to $\mathbb{S}^{3}\left(R_{1}\right) \times \mathbb{S}^{3}\left(R_{2}\right)$. So we have

$$
\eta\left(Z_{l_{0}, l_{1}, l_{2}}\right)=\eta\left(l_{0} \xi+l_{1} X_{1}+l_{2} X_{2}\right)=l_{0}+l_{1}\left(\left|z_{0}\right|^{2}-\left|z_{1}\right|^{2}\right)+l_{2}\left(\left|z_{2}\right|^{2}-\left|z_{3}\right|^{2}\right),
$$

with $l_{0}>0$. The desired result now follows if we use the expression (29) for the radii.

For $\left(l_{0}, l_{1}, l_{2}\right)=(1,0,0)$, we obtain the original Kähler structure on the base $\mathbb{C P}^{1} \times \mathbb{C P}^{1}$, with Kähler metric $k_{1} \omega_{1}+k_{2} \omega_{2}$ where $\omega_{i}$ is the standard Fubini-Study Kähler form on the corresponding $\mathbb{C P}^{1}$ factor. The scalar curvature of this metric is constant, and therefore, the Reeb vector field $\xi$ on $M_{k_{1}, k_{2}}^{1,1}$ is strongly extremal. Moreover, by the openness theorem [13, there exist an open neighborhood of $\xi$ in $\kappa\left(\mathcal{D}_{k_{1}, k_{2}}, J\right)$ that is entirely contained in the extremal set $\mathfrak{e}\left(\mathcal{D}_{k_{1}, k_{2}}, J\right)$. It would be of interest to determine how big a neighborhood this can be, and how it compares to the entire Sasaki cone. Although each $M_{k_{1}, k_{2}}^{1,1}$ carries a Sasakian structure $\mathcal{S}_{k_{1}, k_{2}}$ with an extremal Sasakian metric of constant scalar curvature, the only one with a Sasaki-Einstein metric is $M_{1,1}^{1,1}$. This follows from the fact that $c_{1}\left(\mathcal{D}_{k_{1}, k_{2}}\right)=$ $2\left(k_{2}-k_{1}\right) x$, where $x$ is the positive generator of $H^{2}\left(\mathbb{S}^{2} \times \mathbb{S}^{3}, \mathbb{Z}\right)$.

\section{APPENDiX}

We begin describing a graphical procedure that generalizes the now well-known Brieskorn graphs (see Theorem 10.3.5 and Remark 10.3.1 of [7] for a detailed discussion). 
To a given link $L_{f}(\mathbf{w}, d)=L_{f}(\mathbf{u}, \mathbf{v})$ defined by a weighted homogeneous polynomial $f$, of weight vector $\mathbf{w}$ and fractional weight vector $(\mathbf{u}, \mathbf{v})$, we associate a graph as follows:

Definition 7.4. Consider the following Rational Brieskorn Polytope

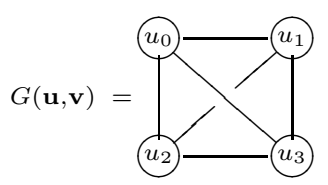

where we think of $G(\mathbf{u}, \mathbf{v})$ as a tetrahedron, and

(1) we label the vertices with pairs $\left(u_{i}, \alpha_{i}\right), i=0,1,2,3$, where $\alpha_{i}=1-1 / v_{j}-$ $1 / v_{k}-1 / v_{l}$ for a set of distinct indices $\{i, j, k, l\}$,

(2) we label the edges with the numbers $\alpha_{i j}=\operatorname{gcd}\left(u_{i}, u_{j}\right) / v_{j} v_{j}$,

(3) we label the faces with numbers $\alpha_{i j k}=\operatorname{gcd}\left(u_{i}, u_{j}, u_{k}\right) / v_{i} v_{j} v_{k}$,

(4) we label the interior of the tetrahedron with the pair

$$
(t, \tau) \equiv\left(\frac{u_{0} u_{1} u_{2} u_{3}}{v_{0} v_{1} v_{2} v_{3} \operatorname{lcm}\left(u_{1}, u_{1}, u_{2}, u_{3}\right)},-1+\frac{1}{v_{0}}+\frac{1}{v_{1}}+\frac{1}{v_{2}}+\frac{1}{v_{3}}\right) .
$$

Furthermore,

(5) we define the reduced indices $m_{i}$ to be the factor of $u_{i}$ that is not a factor of any of the $u_{j}$ s associated to the remaining 3 vertices,

(6) we define numbers $g_{i}$ so that $2 g_{i}+\alpha_{i}$ is computed from the face opposite to the vertex $\left(u_{i}, \alpha_{i}\right)$ as the product of the edge numbers divided by the face number minus the sum of the edge numbers,

(7) we define $\kappa$ so that $\kappa+\tau-t$ is the sum of the six edge numbers minus the sum of four products of edge numbers divided by the face numbers, one such term for each face.

Using this associated graph, we can reformulate Orlik's Conjecture 5.7 in dimension five as follows:

Conjecture 7.5. The second homology group over $\mathbb{Z}$ of a five dimensional link $L_{f}(\mathbf{u}, \mathbf{v})$ is given by

$$
H_{2}(L(\mathbf{a}), \mathbb{Z})=\mathbb{Z}^{\kappa} \oplus\left(\mathbb{Z} / m_{0}\right)^{2 g_{0}} \oplus\left(\mathbb{Z} / m_{1}\right)^{2 g_{1}} \oplus\left(\mathbb{Z} / m_{2}\right)^{2 g_{2}} \oplus\left(\mathbb{Z} / m_{3}\right)^{2 g_{3}},
$$

where $\kappa, m_{i}, g_{i}$ are the integers given in Definition 7.4.

That $\kappa$ is the second Betti number of the link was known long ago [31. There remains to describe the torsion.

Before going any further in this direction, we need to reformulate Kollár's Theorem 6.1 for 5 dimensional links $L_{f}(\mathbf{w}, d)$. First, let us recall the following two simple conditions of quasi-smoothness for curves [22] and surfaces [23]:

Proposition 7.6. 22] Let $C_{d} \subset \mathbb{C P}^{2}\left(w_{0}, w_{1}, w_{2}\right)$ be a curve of degree d defined by a homogeneous polynomial $f$. Assume that $d>a_{i}$, $a_{i}$ integer, $i=0,1,2$. Then $C_{d}$ is quasi-smooth if for all $i \in\{0,1,2\}$ the following conditions are satisfied:

(1) the polynomial $f$ has a monomial $z_{i}^{a_{i}} z_{j}$ for some $j$ (here we allow $i=j$ ) of degree $d$

(2) the polynomial $f$ has a monomial of degree $d$ which does not involve $z_{i}$. 
Proposition 7.7. 22, 23, A general hypersurface $X_{d} \subset \mathbb{C P}\left(w_{0}, w_{1}, w_{2}, w_{3}\right)$ defined by a homogeneous polynomial $f$ is quasi-smooth if, and only if, all of the following three conditions hold

(1) For each $i=0, \ldots, 3$ there is an index $j$ such that $f$ has a monomial $z_{i}^{m_{i}} z_{j} \in H^{0}(\mathbb{C P}(\mathbf{w}), \mathcal{O}(d))$. Here $j=i$ is possible.

(2) If $\operatorname{gcd}\left(w_{i}, w_{j}\right)>1$ then $f$ has a monomial $z_{i}^{b_{i}} z_{j}^{b_{j}} \in H^{0}(\mathbb{C P}(\mathbf{w}), \mathcal{O}(d))$.

(3) For every $i, j$ either $f$ has a monomial $z_{i}^{b_{i}} z_{j}^{b_{j}} \in H^{0}(\mathbb{C P}(\mathbf{w}), \mathcal{O}(d))$, or $f$ has monomials $z_{i}^{c_{i}} z_{j}^{c_{j}} z_{k}$ and $z_{i}^{d_{i}} z_{j}^{d_{j}} z_{l} \in H^{0}(\mathbb{C P}(\mathbf{w}), \mathcal{O}(d))$ with $\{k, l\} \neq\{i, j\}$.

We are then ready for the following proposition.

Proposition 7.8. Let $L_{f}(\mathbf{w}, d)$ be a link defined by a quasi-smooth weighted homogeneous polynomial $f=f\left(z_{0}, z_{1}, z_{2}, z_{3}\right)$, with weights $\mathbf{w}=\left(w_{0}, w_{1}, w_{2}, w_{3}\right)$, and of degree d. Let $L_{f} \rightarrow X_{f} \subset \mathbb{C P}^{3}\left(w_{0}, w_{1}, w_{2}, w_{3}\right)$ be the associated Seifert fibration. Consider the sets

$$
D_{i}=X_{f} \cap\left\{z_{i}=0\right\} \subset \mathbb{C P}^{2}\left(w_{0}, \ldots, \hat{w}_{i}, \ldots, w_{3}\right) .
$$

Note that $D_{i}$ need not be an orbifold, so pick $i \in A \subset \Omega=\{0,1,2,3\}$ such that $D_{i}$ is a quasi-smooth (orbifold) Riemann surface. Then $D_{i}$ can be singular only if $\operatorname{gcd}\left(w_{0}, \ldots, \hat{w}_{i}, \ldots, w_{3}\right)=1$, and we have that

$$
H_{2}\left(L_{f}, \mathbb{Z}\right)=\mathbb{Z}^{\kappa} \oplus \sum_{i}\left(\mathbb{Z} / m_{i}\right)^{2 g\left(D_{i}\right)},
$$

where $\kappa=b_{2}\left(L_{f}\right)=b_{2}\left(X_{f}\right)-1$, and the ramification indices are given by $m_{i}=$ $\operatorname{gcd}\left\{w_{0}, \ldots, \hat{w}_{i}, \ldots, w_{3}\right\}$. In particular, let us set $d_{i}=d / m_{i}$, and let us normalize the weight vector by $\tilde{\mathbf{w}}^{i}=\frac{1}{m_{i}}\left(w_{0}, \ldots, \hat{w}_{i}, \ldots, w_{3}\right)$. Then $D_{i} \subset \mathbb{C P}^{2}\left(\tilde{\mathbf{w}}^{i}\right)$ is an orbifold Riemann surface of degree $d_{i}$ embedded in the weighted projective 2-space with weights $\tilde{\mathbf{w}}^{i}$, and its genus $g\left(D_{i}\right)$ is given by the formula [35, 33.

$$
g\left(\Sigma_{(\tilde{\mathbf{w}}, d)}\right)=\frac{1}{2}\left(\frac{d^{2}}{\tilde{w}_{0} \tilde{w}_{1} \tilde{w}_{2}}-d \sum_{i<j} \frac{\operatorname{gcd}\left(\tilde{w}_{i}, \tilde{w}_{j}\right)}{\tilde{w}_{i} \tilde{w}_{j}}+\sum_{i} \frac{\operatorname{gcd}\left(d, \tilde{w}_{i}\right)}{\tilde{w}_{i}}-1\right) .
$$

Proof. We only need to show that if $D_{i}$ is singular, then we must have $m_{i}=1$ so that $D_{i} \subset X_{f}^{r e g}$ lies inside the smooth part of $X_{f}$. This will follow from the quasi-smoothness condition on $f$.

Suppose then that $m_{0}=\operatorname{gcd}\left(w_{1}, w_{2}, w_{3}\right)>1$, and that $D_{0}$ is singular. We shall derive a contradiction.

Since $m_{0}>1$, we have $\operatorname{gcd}\left(w_{i}, w_{j}\right)>1$ for all $1 \leq i<j \leq 3$. Since $X_{f} \subset$ $\mathbb{C P}^{3}\left(w_{0}, w_{1}, w_{2}, w_{3}\right)$ is quasi-smooth, $f(\mathbf{z})$ must contain the following monomial terms (other than the terms involving $z_{0}$ ):

$$
z_{1}^{a_{1}} z_{j}, \quad z_{2}^{a_{2}} z_{k}, \quad z_{3}^{a_{3}} z_{l}, \quad j, k, l \in\{1,2,3\},
$$

and

$$
z_{1}^{\alpha} z_{2}^{\beta}, \quad z_{2}^{\gamma} z_{3}^{\delta}, \quad z_{3}^{\tau} z_{1}^{\rho} .
$$

Note that, for example, $z_{1}^{a_{1}} z_{0}$ is not possible as this would mean that

$$
d=w_{0}+w_{1} a_{1}
$$

and $m_{0}$ divides $d$ and $w_{1}$ but not $w_{0}$. Hence, the polynomial $g\left(z_{1}, z_{2}, z_{3}\right)=$ $f\left(0, z_{1}, z_{2}, z_{3}\right)$ will contain these six monomial terms. Note that the degree of $g$ is $\tilde{d}=d / m_{0}$, and it has weights $\tilde{w}_{i}=w_{i} / m_{0}$. By Proposition 7.6, it is quasi-smooth. This contradicts the fact that $D_{0}$ is assumed to be singular. 
7.1. Proof of Orlik's Conjecture in Dimension 5. In order to prove Conjecture 7.5. we only need to show that the $\left(\kappa, m_{i}, g_{i}\right) \mathrm{s}$ of Definition 7.4 are the same as the ones in Proposition 7.8. We need to pass between the $(\mathbf{u}, \mathbf{v})$-data and the $(\mathbf{w}, d)$ data.

It suffices to prove the needed statement for $i=0$. We first observe that $\left(m_{0}, k_{0}\right)=\left(c_{123}, k_{123}\right)$. In terms of the $(\mathbf{u}, \mathbf{v})$-data, we have

$$
m_{0}=\frac{u_{0} \operatorname{gcd}\left(u_{0}, u_{1}, u_{2}\right) \operatorname{gcd}\left(u_{0}, u_{1}, u_{2}\right) \operatorname{gcd}\left(u_{0}, u_{2}, u_{3}\right) \operatorname{gcd}\left(u_{0}, u_{1}, u_{3}\right)}{\operatorname{gcd}\left(u_{0}, u_{1}, u_{2}, u_{3}\right) \operatorname{gcd}\left(u_{0}, u_{1}\right) \operatorname{gcd}\left(u_{0}, u_{2}\right) \operatorname{gcd}\left(u_{0}, u_{3}\right)},
$$

and

$$
\begin{aligned}
2 g_{0}= & -1+\left(\frac{1}{v_{1}}+\frac{1}{v_{2}}+\frac{1}{v_{3}}\right)-\left(\frac{u_{1} u_{2}}{v_{1} v_{2} \operatorname{lcm}\left(u_{1}, u_{2}\right)}+\frac{u_{1} u_{3}}{v_{1} v_{3} \operatorname{lcm}\left(u_{1}, u_{3}\right)}+\frac{u_{2} u_{3}}{v_{2} v_{3} \operatorname{lcm}\left(u_{2}, u_{3}\right)}\right) \\
& +\frac{u_{1} u_{2} u_{3}}{v_{1} v_{2} v_{3} \operatorname{lcm}\left(u_{1}, u_{2}, u_{3}\right)} .
\end{aligned}
$$

We recall now that

$$
u_{i}=\frac{d}{\operatorname{gcd}\left(d, w_{i}\right)}, \quad v_{i}=\frac{w_{i}}{\operatorname{gcd}\left(d, w_{i}\right)}, \quad \frac{u_{i}}{v_{i}}=\frac{d}{w_{i}} .
$$

Let us begin with $2 g_{0}$. The first term is no problem as

$$
\frac{1}{v_{i}}=\frac{\operatorname{gcd}\left(d, w_{i}\right)}{w_{i}}
$$

as needed.

Since $f$ is quasi-smooth, $\operatorname{gcd}\left(w_{i}, w_{j}\right)$ must divide the degree for all $i<j$. By this, we have the following

$$
\begin{aligned}
\frac{u_{i} u_{j}}{v_{i} v_{j} \operatorname{lcm}\left(u_{i}, u_{j}\right)} & =\frac{\operatorname{gcd}\left(u_{i}, u_{j}\right)}{v_{i} v_{j}}=\frac{\operatorname{gcd}\left(u_{i}, u_{j}\right) \operatorname{gcd}\left(d, w_{j}\right) \operatorname{gcd}\left(d, w_{i}\right)}{w_{i} w_{j}} \\
& =\frac{\operatorname{gcd}\left(u_{i}\left(\operatorname{gcd}\left(d, w_{j}\right) \operatorname{gcd}\left(d, w_{i}\right)\right), u_{j}\left(\operatorname{gcd}\left(d, w_{j}\right) \operatorname{gcd}\left(d, w_{i}\right)\right)\right)}{w_{i} w_{j}} \\
& =\frac{\operatorname{gcd}\left(d \operatorname{gcd}\left(d, w_{j}\right), d \operatorname{gcd}\left(d, w_{i}\right)\right)}{w_{i} w_{j}}=\frac{d \operatorname{gcd}\left(\operatorname{gcd}\left(d, w_{j}\right), \operatorname{gcd}\left(d, w_{i}\right)\right)}{w_{i} w_{j}} \\
& =\frac{d \operatorname{gcd}\left(w_{i}, w_{j}\right)}{w_{i} w_{j}}
\end{aligned}
$$

where the last equality holds because gcd $\left(w_{i}, w_{j}\right)$ divides $d$. Alternatively (an argument that is simpler), we could get the same result by showing that if $\operatorname{gcd}\left(w_{i}, w_{j}\right) \mid d$, then

$$
\operatorname{lcm}\left(\frac{d}{\operatorname{gcd}\left(d, w_{i}\right)}, \frac{d}{\operatorname{gcd}\left(d, w_{i}\right)}\right)=\frac{d}{\operatorname{gcd}\left(w_{i}, w_{j}\right)} .
$$

The last term is handled similarly,

$$
\frac{u_{1} u_{2} u_{3}}{v_{1} v_{2} v_{3} \operatorname{lcm}\left(u_{1}, u_{2}, u_{3}\right)}=\frac{d^{2} \operatorname{gcd}\left(w_{1}, w_{2}, w_{3}\right)}{w_{1} w_{2} w_{3}}
$$

because under the assumption that $\operatorname{gcd}\left(w_{i}, w_{j}\right) \mid d$ for all $i \neq j$, we have that

$$
\operatorname{lcm}\left(\frac{d}{\operatorname{gcd}\left(d, w_{i}\right)}, \frac{d}{\operatorname{gcd}\left(d, w_{i}\right)}, \frac{d}{\operatorname{gcd}\left(d, w_{k}\right)}\right)=\frac{d}{\operatorname{gcd}\left(w_{i}, w_{j}, w_{k}\right)} .
$$


Combining all of these terms, we get that

$$
2 g_{0}=-1+\sum_{i=1}^{3} \frac{\operatorname{gcd}\left(d, w_{i}\right)}{w_{i}}-d \sum_{1 \leq i<j \leq 3} \frac{\operatorname{gcd}\left(w_{i}, w_{j}\right)}{w_{i} w_{j}}+\frac{d^{2} \operatorname{gcd}\left(w_{1}, w_{2}, w_{3}\right)}{w_{1} w_{2} w_{3}} .
$$

If we now re-scale

$$
\tilde{\mathbf{w}}=\frac{\left(w_{1}, w_{2}, w_{3}\right)}{\operatorname{gcd}\left(w_{1}, w_{2}, w_{3}\right)}, \quad \tilde{d}=\frac{d}{\operatorname{gcd}\left(w_{1}, w_{2}, w_{3}\right)},
$$

we see that for $0 \leq i<j<k \leq 3$ we have that $k_{i j k}=2 g\left(\Sigma_{(\tilde{\mathbf{w}}, \tilde{d})}\right)$, as desired.

It remains to show that $m_{0}=\operatorname{gcd}\left(w_{1}, w_{2}, w_{3}\right)$, which we shall leave as an exercise.

The conjecture follows now by Proposition 7.8 . This proposition shows that when any of the $m_{i}>1$, then the corresponding $g_{i}$ is the genus of a quasi-smooth curve $D_{i}$, and therefore, $2 g_{i}$ must not only be integral, but also equal to twice that genus as it was shown. When $m_{i}=1$, it does not matter what $2 g_{i}$ is as it does not enter into the torsion formula in either Kollár's theorem or in Orlik's Algorithm.

\section{REFERENCES}

[1] A. Bangaya \& P. Molino, Géométric des formes de contact complètement intégrables de type toriques, Séminaire G. Darboux de Géométrie et Topologie Différentielle, 1991-1992, Montpellier.

[2] D. Barden, Simply connected five-manifolds, Ann. of Math. (2) 82 (1965), pp. 365-385. MR 32 \#1714.

[3] C.P. Boyer \& K. Galicki, A note on toric contact geometry, J. Geom. Phys. 35 (2000), 4, pp. 288-298. MR 2001h:53124.

[4] _ Einstein metrics on rational homology spheres, J. Diff. Geom. (3) 74 (2006), pp. 353-362. MR MR2269781.

[5] __ New Einstein metrics in dimension five, J. Diff. Geom. (3) 57 (2001), pp. 443-463. MR 2003b:53047.

[6] _ On Sasakian-Einstein geometry, Internat. J. Math. (7) 11 (2000), pp. 873-909. MR 2001k:53081.

[7] _ Sasakian Geometry, Oxford Mathematical Monographs, Oxford University Press, to appear, 2008.

[8] - Sasakian geometry, hypersurface singularities, and Einstein metrics, Rend. Circ. Mat. Palermo (2) Suppl. (2005), 75, suppl., pp. 57-87. MR 2152356.

[9] C.P. Boyer, K. Galicki \& J. Kollár, Einstein metrics on spheres, Ann. Math. (2) 162 (2005), no. 1, pp. 557-580. MR 2178969(2006j:53058).

[10] C.P. Boyer, K. Galicki, J. Kollár \& E. Thomas, Einstein metrics on exotic spheres in dimensions 7, 11, and 15, Experiment. Math. 14 (2005), no. 1, pp. 59-64. MR 2146519 (2006a:53042).

[11] C.P. Boyer, K. Galicki \& P. Matzeu, On eta-Einstein Sasakian geometry, Comm. Math. Phys. (1) 262 (2006), pp. 177-208. MR 2200887.

[12] C.P. Boyer, K. Galicki \& L. Ornea, Constructions in Sasakian Geometry, preprint, arXiv:math.DG/0602233, to appear in Mathematische Zeitscrift (2007).

[13] C.P. Boyer, K. Galicki \& S.R. Simanca, Canonical Sasakian metrics, Comm. Math. Phys (to appear), math.DG/0604325

[14] E. Calabi, Extremal Kähler metrics II, in Differential geometry and complex analysis (I. Chavel \& H.M. Farkas eds.), Springer-Verlag, 1985, pp. 95-114.

[15] K. Cho, A. Futaki \& H. Ono, Uniqueness and examples of compact toric Sasaki-Einstein metrics, preprint; arXiv:math.DG/0701122 (2007).

[16] A. Futaki, An obstruction to the existence of Einstein Kähler metrics, Invent. Math., 73 (1983), pp. 437-443. MR 84j:53072.

[17] A. Futaki, H. Ono \& G. Wang, Transverse Kähler geometry of Sasaki manifolds and toric Sasaki-Einstein manifolds, preprint arXiv:math.DG/0607586, (2006). 
[18] J.P. Gauntlett, D. Martelli, J. Sparks \& W. Waldram, Sasaki-Einstein metrics on $S^{2} \times S^{3}$, Adv. Theor. Math. Phys., 8 (2004), pp. 711-734.

[19] J.P. Gauntlett, D. Martelli, J. Sparks \& S.-T. Yau, Obstructions to the existence of SasakiEinstein metrics, Comm. Math. Phys. (3) 273 (2007), pp. 803-827.

[20] A. Ghigi \& J. Kollár, Kähler-Einstein metrics on orbifolds and Einstein metrics on Spheres, Comment. Math. Helvetici (to appear), arXiv:math.DG/0507289

[21] G. Grantcharov \& L. Ornea, Reduction of Sasakian manifolds, J. Math. Phys. (8) 42 (2001), pp. 3809-3816. MR 1845220 (2002e:53060).

[22] A.R. Iano-Fletcher, Working with weighted complete intersections, Explicit birational geometry of 3-folds, London Math. Soc. Lecture Note Ser. 281, pp. 101-173, Cambridge Univ. Press, Cambridge, (2000).

[23] J.M. Johnson \& J. Kollár, Kähler-Einstein metrics on log del Pezzo surfaces in weighted projective 3-spaces, Ann. Inst. Fourier (Grenoble) (1) 51 (2001), pp. 69-79. MR 2002b:32041.

[24] J. Kollár, Circle actions on simply connected 5-manifolds, Topology (3) 45 (2006), pp. 643-671. MR 2218760.

[25] - Einstein metrics on five-dimensional Seifert bundles, J. Geom. Anal. (3) 15 (2005), pp. 445-476. MR 2190241.

[26] — Positive Sasakian structures on 5-manifolds, these Proceedings, Eds. Galicki \& Simanca, Birkhauser.

[27] C. LeBrun \& S.R. Simanca, On the Kähler Classes of Extremal Metrics, Geometry and Global Analysis (Sendai, Japan 1993), First Math. Soc. Japan Intern. Res. Inst. Eds. Kotake, Nishikawa \& Schoen.

[28] E. Lerman, Contact toric manifolds, J. Symp. Geom. (4) 1 (2002), pp. 785-828, MR 2039 164.

[29] D. Martelli, J. Sparks \& S.-T. Yau, Sasaki-Einstein manifolds and volume minimisation, arXiv:hep-th/0603021 preprint 2006.

[30] J.W. Milnor, Singular points of complex hypersurfaces, Annals of Mathematics Studies 61, Princeton University Press, Princeton, N.J., 1968. MR 39 \#969.

[31] J. Milnor \& P. Orlik, Isolated singularities defined by weighted homogeneous polynomials, Topology 9 (1970), pp. 385-393. MR 45 \#2757.

[32] P. Orlik, On the homology of weighted homogeneous manifolds, Proceedings of the $2^{\text {nd }}$ Conference on Compact Transformation Groups (Univ. of Mass., Amherst, Mass., 1971), Part I, Lect. Notes Math. 298, Springer-Verlag (Berlin), 1972, pp. 260-269. MR 55 \#3312.

[33] _ Seifert manifolds, Lect. Notes Math. 291, Springer-Verlag (Berlin), 1972, MR 54 \#13950.

[34] P. Orlik \& R.C. Randell, The monodromy of weighted homogeneous singularities, Invent. Math. (3) 39 (1977), pp. 199-211. MR 57 \#314.

[35] P. Orlik \& P. Wagreich, Isolated singularities of algebraic surfaces with $C^{*}$ action, Ann. of Math. (2) 93 (1971), pp. 205-228. MR 44 \#1662.

[36] R.C. Randell, The homology of generalized Brieskorn manifolds, Topology (4) 14 (1975), pp. 347-355. MR 54 \#1270.

[37] P. Rukimbira, Chern-Hamilton's conjecture and K-contactness, Houston J. Math. (4) 21 (1995), pp. 709-718. MR 96m:53032.

[38] S.R. Simanca, Canonical metrics on compact almost complex manifolds, Publicações Matemáticas do IMPA, IMPA, Rio de Janeiro, 2004. 97 pp.

[39] - Heat Flows for Extremal Kähler Metrics, Ann. Scuola Norm. Sup. Pisa CL. Sci., 4 (2005), pp. 187-217.

[40] — Precompactness of the Calabi Energy, Internat. J. Math., 7 (1996) pp. 245-254.

[41] _ Strongly Extremal Kähler Metrics, Ann. Global Anal. Geom. 18 (2000), no. 1, pp. $29-46$.

[42] S.R. Simanca \& L.D. Stelling, Canonical Kähler classes. Asian J. Math. 5 (2001), no. 4, pp. $585-598$.

[43] S. Smale, On the structure of 5-manifolds, Ann. of Math. (2) 75 (1962), pp. 38-46. MR 25 \#4544.

[44] T. Takahashi, Deformations of Sasakian structures and its application to the Brieskorn manifolds, Tôhoku Math. J. (2) 30 (1978), no. 1, pp. 37-43. MR 81e:53024.

[45] M.Y. Wang \& W. Ziller, Einstein metrics on principal torus bundles, J. Diff. Geom. (1) 31 (1990), pp. 215-248. MR 91f:53041. 
[46] S.S.-T. Yau and Y. Yu, Classification of 3-dimensional isolated rational hypersurface singularities with $\mathbb{C}^{*}$-action, Rocky Mountain J. Math. (5) 35 (2005), pp. 1795-1809. MR 2206037(2006j:32034).

Department of Mathematics and Statistics, University of New Mexico, Albuquerque, N.M. 87131

E-mail addresses: cboyer@math.unm.edu, santiago@math.unm.edu 